\title{
The long-wavelength emission of interstellar PAHs: characterizing the spinning dust contribution
}

\begin{abstract}
N. Ysard and L. Verstraete
Institut d'Astrophysique Spatiale, UMR 8617, Université Paris-Sud, 91405 Orsay, France

e-mail: nathalie.ysard@ias.u-psud.fr

Received 17 June 2009 / Accepted 24 September 2009

ABSTRACT

Context. The emission of cold dust grains at long wavelengths will soon be observed by the Planck and Herschel satellites and will provide new constraints on the nature of interstellar dust. In particular, the microwave galactic anomalous foreground detected between 10 to $90 \mathrm{GHz}$, proposed as coming from small spinning grains (PAHs), should help to define these species better. Moreover, understanding the fluctuations of the anomalous foreground quantitatively over the sky is crucial for CMB studies.

Aims. We focus on the long-wavelength emission of interstellar PAHs in their vibrational and rotational transitions. We present here the first model that coherently describes the PAH emission from the near-IR to microwave range.

Methods. We take quantum effects into account to describe the rotation of PAHs and compare our results to current models of spinning dust to assess the validity of the classical treatment used. Between absorptions of stellar photons, we followed the rovibrational radiative cascade of PAHs. We used the exact-statistical method of Draine \& Li to derive the distribution of PAH internal energy and followed a quantum approach for the rotational excitation induced by vibrational (IR) transitions. We also examined the influence of the vibrational relaxation scheme and of the low-energy cross-section on the PAH emission. We study the emissivity of spinning PAHs in a variety of physical conditions (radiation field intensity and gas density), search for specific signatures in this emission that can be looked for observationally, and discuss how the anomalous foreground may constrain the PAH size distribution.

Results. Simultaneously predicting the vibrational and rotational emission of PAHs, our model can explain the observed emission of the Perseus molecular cloud from the IR to the microwave range with plausible PAH properties. We show that for $\lambda \geq 3 \mathrm{~mm}$ the PAH vibrational emission no longer scales with the radiation field intensity $\left(G_{0}\right)$, unlike the mid-IR part of the spectrum (which scales with $G_{0}$ ). This emission represents less than $10 \%$ of the total dust emission at $100 \mathrm{GHz}$. Similarly, we find the broadband emissivity of spinning PAHs per carbon atom to be rather constant for $G_{0} \leq 100$ and for proton densities $n_{\mathrm{H}}<100 \mathrm{~cm}^{-3}$. In the diffuse ISM, photon exchange and gas-grain interactions play comparable roles in exciting the rotation of PAHs, and the emissivity of spinning PAHs is dominated by the contribution of small species (bearing less than $100 \mathrm{C}$ atoms). We show that the classical description of rotation used in previous works is a good approximation and that unknowns in the vibrational relaxation scheme and low-energy cross-section affect the PAH rotational emissivity around $30 \mathrm{GHz}$ by less than $15 \%$.

Conclusions. The contrasted behaviour of the PAH vibrational and rotational emissivities with $G_{0}$ provides a clear prediction that can be tested against observations of anomalous and dust mid-IR emissions: this is the subject of a companion paper. Comparison of these emissions complemented with radio observations $(21 \mathrm{~cm}$ or continuum) will provide constraints on the fraction of small species and the electric dipole moment of interstellar PAHs.
\end{abstract}

Key words. ISM: general - dust, extinction - radiation mechanisms: general

\section{Introduction}

The mid-IR spectrum of the interstellar medium (ISM) shows prominent bands from 3 to $17 \mu \mathrm{m}$, which account for one third of the energy emitted by interstellar dust. Such bands are emitted by very small (subnanometric-sized) dust particles during internal energy fluctuations triggered by the absorption of a stellar photon (Sellgren 1984). The positions of these bands suggest there are aromatic, hydrogenated cycles in these grains. Léger \& Puget (1984) and Allamandola et al. (1985) proposed polycyclic aromatic hydrocarbons (PAHs) as the carriers of these bands. Despite two decades of experimental and theoretical efforts, the match (band positions and intensities) between data only available on small species and observations still remains elusive, as illustrated recently by the work of Peeters et al. (2002) and Kim \& Saykally (2002). Given their important role in the ISM (e.g., gas heating, Habart et al. 2001; UV extinction, Joblin et al. 1992), it is necessary to find other ways to constrain the properties of interstellar PAHs.
The Planck and Herschel data will soon trace the emission of cold interstellar grains in the interstellar medium. Due to their small size, PAHs are heated sporadically (every few months in the diffuse ISM) by absorption of stellar photons and have a high probability of being in low-energy states (Draine \& Li 2001). Interstellar PAHs may thus contribute significantly to the emission at long wavelength $(\lambda>1 \mathrm{~mm})$. In this context, an unexpected emission excess called anomalous foreground, correlated to dust emission, has been discovered between 10 and $90 \mathrm{GHz}$ (Leitch et al. 1997; de Oliveira-Costa et al. 2002). In this spectral range, several galactic emission components (synchrotron, freefree, and thermal dust) contribute with a comparable magnitude, and only recently has the anomalous foreground been separated in WMAP data (Miville-Deschênes et al. 2008). Spinning, small dust grains were first proposed by Draine \& Lazarian (1998) (hereafter DL98) as a possible origin to this anomalous component. Since then, analysis of observations has suggested that the anomalous foreground is correlated to small grain emission (Lagache 2003; Casassus et al. 2006). 
In this paper, we study the emission of PAHs with particular emphasis on the long-wavelength part of the spectrum. In this spectral range, the emission is dominated by species in lowenergy states for which each photon exchange represents a large energy fluctuation. To derive the internal energy distribution of PAHs, we use here the exact statistical method described in Draine \& Li (2001). We include low-frequency bands to the vibrational mode spectrum of interstellar PAHs and examine the influence of the internal vibrational redistribution hypothesis (see Sect. 3) often used to describe their vibrational relaxation. We describe the rotation of PAHs with a quantum approach where specific processes in the rovibrational relaxation are naturally included (molecular recoil after photon emission; rovibrational transitions that leave the angular momentum of the molecule unchanged or $Q$ bands). We present in a variety of interstellar phases the rovibrational (IR) and broadband rotational (spinning) emission of PAHs in a consistent fashion. Our model results are compared to observations of the interstellar emission from the IR to the microwave range and show how the size distribution and electric dipole moments of interstellar PAHs may be constrained.

The paper is organized as follows. Section 2 describes the properties of PAHs adopted in this work. Section 3 discusses the internal energy distribution of PAHs and associated rovibrational emission. Sections 4 and 5 discuss the rotational excitation and emission of PAHs. In Sect. 6, we apply our model to the case of a molecular cloud in the Perseus arm. Finally, conclusions and observational perspectives are given in Sect. 7.

\section{The properties of interstellar PAHs}

In the ISM, the stablest PAHs are found to be compact species (Léger et al. 1989; Le Page et al. 2003). Small PAHs are planar, but above some poorly known size threshold (40 to $100 \mathrm{C}$ atoms), interstellar formation routes may favour threedimensional species (bowl- or cage-shaped) containing pentagonal cycles (see Moutou et al. 2000, and references therein). As we see later, small PAHs (containing less than $100 \mathrm{C}$ atoms) dominate the rotational emission, so we assume that interstellar PAHs are planar with a hexagonal $\left(D_{6 \mathrm{~h}}\right)$ symmetry. The PAH radius is thus $a(\AA)=0.9 \sqrt{N_{\mathrm{C}}}$, where $N_{\mathrm{C}}$ is the number of carbon atoms in the grain (Omont 1986). The formula for such molecules is $\mathrm{C}_{6 \mathrm{p}^{2}} \mathrm{H}_{6 \mathrm{p}}$, and their hydrogen-to-carbon ratio is $\frac{\mathrm{H}}{\mathrm{C}}=f_{\mathrm{H}} \sqrt{\frac{6}{N_{\mathrm{C}}}}$, where $f_{\mathrm{H}}$ is the hydrogenation fraction of PAHs. In this work, we assume that PAHs are fully hydrogenated $\left(f_{\mathrm{H}}=1\right)$.

\subsection{Absorption cross-section}

The excitation, cooling, and emission of PAHs depend on their absorption cross-section (Sect. 3), which we describe now. We took the visible-UV cross-section from Verstraete \& Léger (1992) and applied their size-dependent cut-off for electronic transitions in the visible-NIR range. The resulting cross-section compares well to the available data (Joblin et al. 1992). In Appendix A, we discuss the mid-IR bands considered in this work. Each vibrational mode is assumed to be harmonic, and the corresponding band profile has a Drude shape (Draine \& Li 2001). The width $\Delta v$ is inferred from astronomical spectra, and the peak value $\sigma$ is chosen so that the integrated crosssection $\sigma \Delta v$ is equal to the value measured in the laboratory. By adopting the observed bandwidth, we empirically account for the complex molecular relaxation and band broadening (Pech et al. 2002; Mulas et al. 2006) in interstellar PAHs. We adopt here
Table 1. Far-IR rovibrational bands of PAH cations adopted in this work, with the percentage of the total oscillator strength in each band.

\begin{tabular}{|c|c|c|c|c|c|}
\hline $\begin{array}{c}\lambda_{\text {cations }}{ }^{a} \\
(\mu \mathrm{m})\end{array}$ & $\begin{array}{l}v_{\text {cations }} \\
\left(\mathrm{cm}^{-1}\right)\end{array}$ & $\begin{array}{c}\Delta v \\
\left(\mathrm{~cm}^{-1}\right)\end{array}$ & $\begin{array}{c}\sigma_{i} / N_{\mathrm{C}} \\
\left(10^{-20} \mathrm{~cm}^{2}\right) \\
\end{array}$ & $\begin{array}{c}\% \\
\text { (cations) }\end{array}$ & Type $^{b}$ \\
\hline 30.2 & 331 & 300 & $9.6 \times 10^{-3}$ & 69.5 & $\begin{array}{l}2 / 3 \text { ip } \\
1 / 3 \text { op }\end{array}$ \\
\hline 74.1 & 135 & 100 & $9.9 \times 10^{-3}$ & 23.9 & $\begin{array}{l}2 / 3 \mathrm{ip} \\
1 / 3 \mathrm{op}\end{array}$ \\
\hline $4.9 \times N_{\mathrm{C}}$ & $2040 / N_{\mathrm{C}}$ & 100 & $2.7 \times 10^{-3}$ & 6.6 & $\begin{array}{l}2 / 3 \mathrm{ip} \\
1 / 3 \mathrm{op}\end{array}$ \\
\hline
\end{tabular}

${ }^{a}$ Similar bands were obtained for PAH neutrals. ${ }^{b}$ In-plane (ip) or outof-plane (op) character of the band.

the band strengths given in Pech et al. (2002) that were inferred from laboratory data. Different definitions of the PAH IR crosssections have been proposed by Rapacioli et al. (2005), Flagey et al. (2006), and Draine \& Li (2007).

We used the database of Malloci et al. (2007) to define an average broadband cross-section of the far-IR vibrations of PAHs. At frequencies below $500 \mathrm{~cm}^{-1}$, each species features many modes. However, for compact species, modes accumulate in three definite frequency ranges: modes with a frequency of less than $100 \mathrm{~cm}^{-1}$, modes between 100 and $200 \mathrm{~cm}^{-1}$, and modes between 200 and $500 \mathrm{~cm}^{-1}$. We therefore model the far-IR crosssection of compact PAHs with 3 modes (Table 1). The frequency of each mode is the average of all modes falling within the given energy range, weighted by their corresponding integrated crosssections. We find that the frequency of the lowest energy mode depends on the molecular size as $N_{\mathrm{C}}^{-1}$ (see Fig. 1). Conversely, for the two other modes, the average energy is instead independent of the size. The integrated cross-section for these 3 modes was estimated as follows. From the Mallocci database, we first derived the fraction of $\sigma \Delta v$ for each of the 3 modes. Then, we assumed that the total $\sigma \Delta v$ below $500 \mathrm{~cm}^{-1}$ is given by the integral of the absorption cross-section of Schutte et al. (1993): $\sigma_{\text {FIR }}=4.3 \times 10^{-20} \lambda^{-1.24} \mathrm{~cm}^{2}$ per C-atom. The integrated crosssections of each of the 3 modes was finally obtained by multiplying the former value by the $\sigma \Delta v$ fractions inferred from the database.

The parameters of the far-IR bands adopted here are given in Table 1, and the full cross-section is displayed in Fig. 2. We show in Sect. 3 that this set of IR bands provides a good match to observed interstellar spectra. Finally, we note that the in-plane or out-of-plane character of each vibrational band is important because of the different associated weights in the rotational excitation (Sect. 4.1). This character is indicated in Tables A.1 for the mid-IR bands (Socrates 2001). In the case of the far-IR bands, this character is not as well known and we assume that $1 / 3(2 / 3)$ of the oscillator strength come from out-of-plane (in-plane) transitions respectively (Table 1).

\subsection{The rigid rotor model}

While describing the rotation of a molecule, the relevant operator is the total angular momentum $\boldsymbol{J}$, which includes the electrons and nuclei contributions without the spin. We note that $D_{\mathrm{A}}, D_{\mathrm{B}}$, and $D_{\mathrm{C}}$ are the principal axis of inertia. We assumed that PAHs

2 The molecules considered are perylene $\left(\mathrm{C}_{20} \mathrm{H}_{12}\right)$, benzo[g, h, i]perylene $\left(\mathrm{C}_{22} \mathrm{H}_{12}\right)$, coronene $\left(\mathrm{C}_{24} \mathrm{H}_{12}\right)$, bisanthene $\left(\mathrm{C}_{28} \mathrm{H}_{14}\right)$, dibenzo[bc, ef] coronene $\left(\mathrm{C}_{30} \mathrm{H}_{14}\right)$, ovalene $\left(\mathrm{C}_{32} \mathrm{H}_{14}\right)$, circumbiphenyl $\left(\mathrm{C}_{38} \mathrm{H}_{16}\right)$, circumanthracene $\left(\mathrm{C}_{40} \mathrm{H}_{16}\right)$, cirumpyrene $\left(\mathrm{C}_{42} \mathrm{H}_{16}\right)$, and circumovalene $\left(\mathrm{C}_{66} \mathrm{H}_{20}\right)$. 


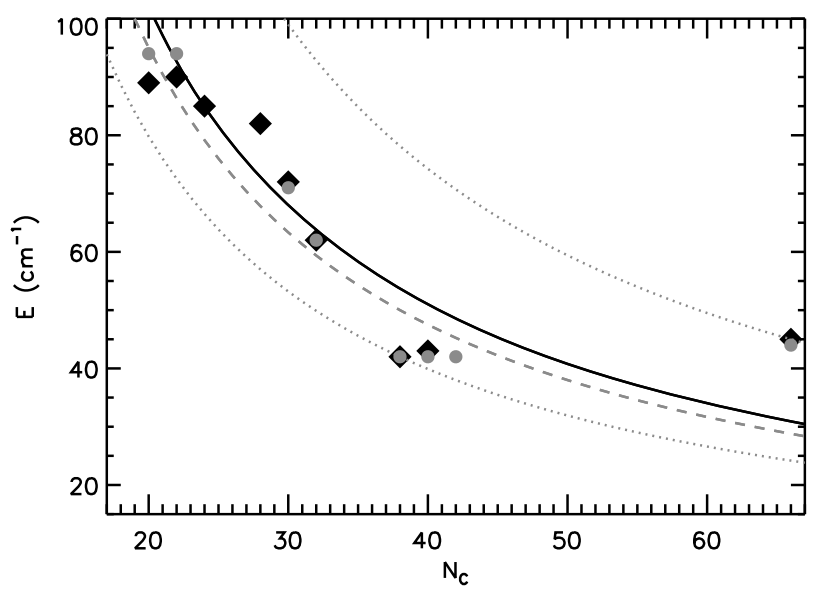

Fig. 1. Frequencies of the first (lowest energy) vibrational mode versus $N_{\mathrm{C}}$ for PAH cations (black diamonds) and PAH neutrals (grey circles) from the Mallocci database ${ }^{2}$ (see text). The solid line is the relationship we adopt between the band position of cations and $N_{\mathrm{C}}$, and the dashed line shows the case of neutrals. The dotted lines show extreme cases for this relationship.

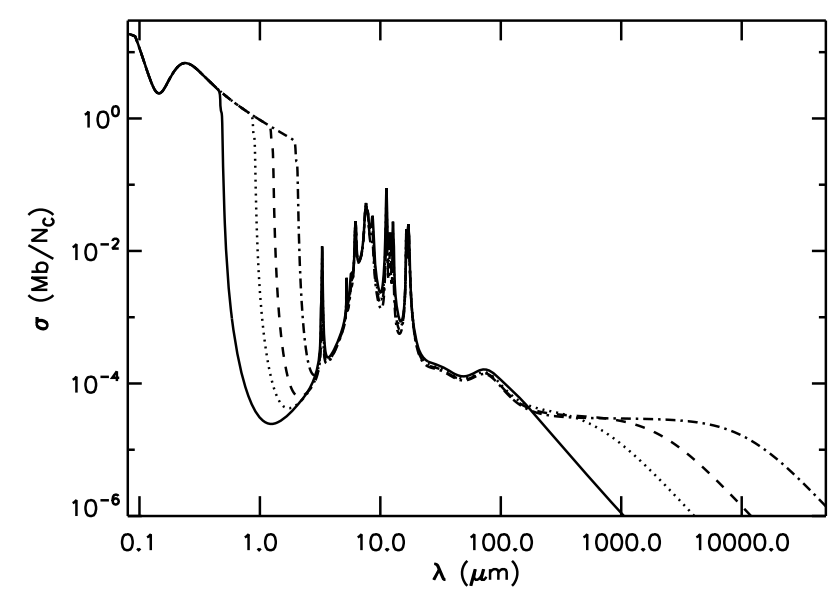

Fig. 2. Absorption cross-section of PAH cations per carbon atom $\left(1 \mathrm{Mb}=10^{-18} \mathrm{~cm}^{2}\right)$. The solid, dotted, dashed, and dot-dashed lines show the cases for $N_{\mathrm{C}}=24,54,96$, and 216 , respectively.

are oblate symmetric top molecules with the axis $D_{\mathrm{C}}$ perpendicular to the plane of the molecule and parallel to $O z$. We called $I_{\mathrm{C}}$ the inertia moment with respect to $D_{\mathrm{C}}$ and $I_{\mathrm{A}}, I_{\mathrm{B}}$ the inertia moments with respect to $D_{\mathrm{A}}$ and $D_{\mathrm{B}}$, which were taken to be parallel to $O x$ and $O y$. The rotational Hamiltonian is then

$H=\frac{J_{x}^{2}}{2 I_{\mathrm{A}}}+\frac{J_{y}^{2}}{2 I_{\mathrm{B}}}+\frac{J_{z}^{2}}{2 I_{\mathrm{C}}}$

where $J_{x}, J_{y}$, and $J_{z}$ are the projections of $\boldsymbol{J}$ along the three inertia axis. Given the large number of carbon atoms in interstellar PAHs, we assume here that they are uniform disks with $I_{\mathrm{A}}=I_{\mathrm{B}}=\frac{M a^{2}}{4}=\frac{I_{\mathrm{C}}}{2}$ (symmetric tops), where $M$ is the molecular mass. With $D_{\mathrm{C}} \| \stackrel{2}{O} z$, we get

$H=\frac{J^{2}}{2 I_{\mathrm{B}}}+J_{z}^{2}\left(\frac{1}{2 I_{\mathrm{C}}}-\frac{1}{2 I_{\mathrm{B}}}\right)$,

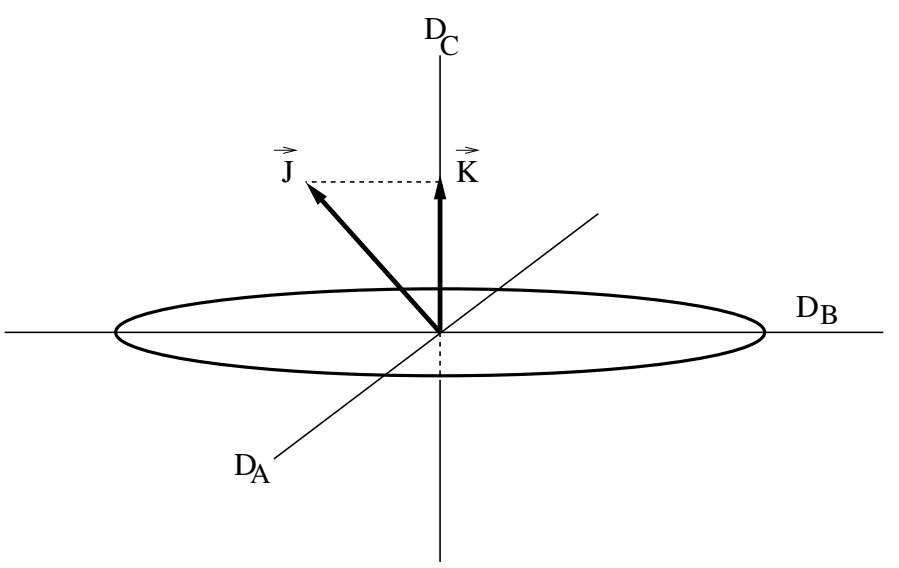

Fig. 3. Symmetric top molecule: $D_{\mathrm{A}}, D_{\mathrm{B}}$, and $D_{\mathrm{C}}$ are the principal axis of inertia and $J$ the total angular momentum of the molecule with $K$ its projection along $D_{\mathrm{C}}$.

and the rotational energy is

$E_{\mathrm{rot}}=B J(J+1)+(C-B) K^{2}$

where $B / h c=\frac{\hbar}{4 \pi c I_{\mathrm{B}}}=7 \mathrm{~N}_{\mathrm{C}}^{-2} \mathrm{~cm}^{-1}$ (neglecting the contributions of $\mathrm{H}$ to the molecular mass) and $C=B / 2$ are the rotational constants associated to $D_{\mathrm{B}}$ and $D_{\mathrm{C}}$. The quantum number $K$ is the absolute value of the $J_{z}$-eigenvalues. For a symmetric top molecule, the selection rules for rovibrational electric dipole transitions are $\Delta J=0, \pm 1$ and $\Delta K=0, \pm 1$. By defining the dipole moment as $\boldsymbol{\mu}=\boldsymbol{\mu}_{z}+\boldsymbol{\mu}_{\mathrm{B}}$, with $\boldsymbol{\mu}_{z}$ along $D_{\mathrm{C}}$ and $\mu_{\mathrm{B}}$ in the molecular plane along $D_{\mathrm{B}}$ (Townes \& Schawlow 1975), two kinds of transitions can be distinguished. First, the parallel transitions with $\Delta K=0$ for which the change of dipole moment in the transition is parallel to the top axis $\left(D_{\mathrm{C}}\right)$ of the molecule. Second, the perpendicular transitions with $\Delta K= \pm 1$, for which the change of dipole moment is perpendicular to $D_{\mathrm{C}}$. Parallel transitions thus correspond to out-of-plane vibrations, whereas perpendicular transitions correspond to in-plane vibrations.

Since the available microwave data are broadband observations $(\lambda / \Delta \lambda$ of the order of a few), we make several simplifying assumptions in the description of the rotational motion of PAHs. We thus assume that the rotational constant $B$ is the same in all vibrational levels, and within the framework of a rigid rotor model, we neglect the centrifugal distortion terms in the energy equation that are usually small for large molecules (Herzberg 1968ab; Lovas et al. 2005).

\subsection{Electric dipole moment}

The rotational emission of PAHs depends on their permanent electric dipole moment, $\mu$. Symmetric $\left(D_{6 \mathrm{~h}}\right)$, neutral, and fully hydrogenated PAHs have $\mu \sim 0$. Spectroscopic analysis of their IR emission bands suggests that interstellar PAHs can hqve a cationic form that is partially hydrogenated (Le Page et al. 2003), and maybe also substituted (Peeters et al. 2002, 2004a). For instance, a PAH having lost one $\mathrm{H}$ atom has $\mu \sim 0.8$ to $1 \mathrm{D}$, and a PAH cation where a $\mathrm{C}$ atom has been substituted by $\mathrm{N}$ would also have $\mu \sim 0.1$ to $1.5 \mathrm{D}$ depending on its size (Pino, private communication). Moreover, it has been proposed that nonplanar PAHs containing pentagonal rings may exist in the ISM (see Moutou et al. 2000, and references therein): such species are known to have large dipole moments as recently measured on coranulene, $\mathrm{C}_{20} \mathrm{H}_{10}, \mu=2 \mathrm{D}$ (Lovas et al. 2005). In this work, 


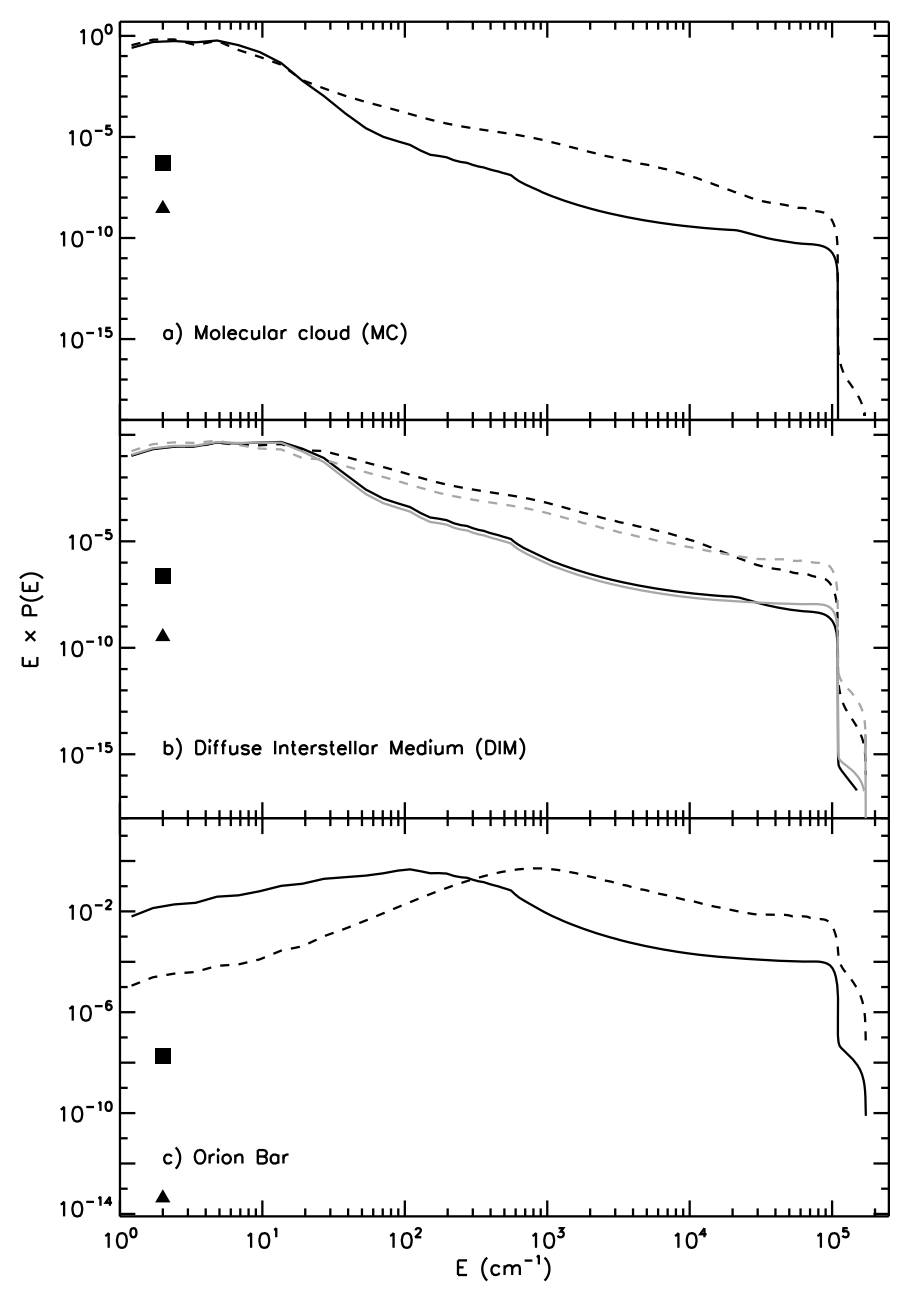

Fig. 4. Internal energy distribution of PAHs with $N_{\mathrm{C}}=24$ (solid line and box for $P(0)$ ) and $N_{\mathrm{C}}=216$ (dashed line and triangle) in the case of a) an MC $\left(G_{0}=10^{-2}\right)$, b) the diffuse ISM $\left(G_{0}=1\right)$ and $\left.\mathbf{c}\right)$ the Orion Bar (the radiation field is the sum of the CMB, the ISRF and a blackbody at $37000 \mathrm{~K}$ corresponding to $G_{0}=14000$ ). To illustrate the effect of the radiation field hardness, we show in b) the case of an Orion Bar type radiation field scaled down to $G_{0}=1$ (grey lines).

we express the electric dipole moment of interstellar PAHs as in DL98:

$\mu\left(N_{\mathrm{C}}\right)=m \sqrt{N_{\mathrm{at}}}+4.3 \times 10^{-2} \sqrt{N_{\mathrm{C}}} Z \simeq m \sqrt{N_{\mathrm{at}}} \quad$ (Debye)

where $N_{\text {at }}$ is the total atoms number in the molecule, $Z$ is its charge, and $m$ a constant. Unless otherwise stated, we will use $m=0.4 \mathrm{D}$.

\section{Internal energy distribution and rovibrational IR emission of isolated interstellar PAHs}

After the absorption of a visible-UV photon, a PAH cools off by emitting IR rovibrational photons. These photons reduce its angular momentum and also may increase it by recoil (a purely quantum effect, see Sect. 4.1). Previous studies have mostly used a thermal description of molecular cooling. In fact, since PAHs spend a large fraction of their time at low internal energies (see Fig. 4), their emission at long wavelengths cannot be considered as a negligible fluctuation compared to their total internal energy. While estimating the emission of PAHs at low temperatures, the validity of a thermal approach is questionable. Because of the rapid energy redistribution between interactions (photon exchange or collision with gas phase species), PAHs rapidly reach thermodynamical equilibrium while isolated (Léger et al. 1989). To describe this situation, we used the exact-statistical method of Draine \& Li (2001) to derive the stationary internal energy distribution of PAHs.

The energy distribution, $P(E)$, depends on (a) the energy density of the exciting radiation field $u_{\mathrm{E}}=4 \pi v I_{v}$ (where $I_{v}$ is the brightness); (b) the absorption cross-section, $\sigma$, of interstellar PAHs; and (c) their rovibrational density of states, $\rho(E)$. For the stellar contribution, we used the local interstellar radiation field (ISRF, Mathis et al. 1983) or a blackbody. We sometimes scale this stellar radiation field with a factor, $G_{0}$, equal to 1 in the case of the Mathis field ${ }^{3}$. Throughout this work, the contribution of the CMB is included in the radiation field. The vibrational absorption cross-section used has been described previously, and for simplicity, we did not include rotational bands. Unless otherwise stated, we took the cross-section of PAH cations. The density of states was obtained by first deriving the vibrational mode spectrum from a Debye model and then applying the algorithm of Beyer \& Swinehart (1973) for each molecular size (see Appendix B).

After each absorption of a stellar photon, we assume that the excitation energy of the PAH is rapidly redistributed among all the vibrational modes (Mulas et al. 2006). This radiationless and isoenergetic process is called internal vibrational redistribution (IVR). The energy distribution is then computed according to the exact-statistical formalism described in Draine \& Li (2001). The energy scale (referenced to the zero point energy) is divided into bins of energy $E_{i}$ and width $\Delta E_{i}$ with $i=0,1, \ldots, M(M=$ 500) with $0 \leq E_{i} \leq 2 E_{\mathrm{L}}$ where $E_{\mathrm{L}}=1.1 \times 10^{5} \mathrm{~cm}^{-1}$ is the Lyman limit. When its energy is below that of the first excited vibrational state, the molecule is in a rotational state. Letting $P_{i}$ be the probability of having a PAH in the energy bin $i$ we have

$$
\frac{\mathrm{d} P_{i}}{\mathrm{~d} t}=\sum_{j \neq i}^{M} T_{j \rightarrow i} P_{j}-\sum_{i \neq j}^{M} T_{i \rightarrow j} P_{i}
$$

where $T_{j \rightarrow i}$ is the transition rate from the state $j$ to the state $i$ for a molecule. We solve this equation in the stationary case. More details can be found in Draine \& Li (2001). Figure 4 shows the energy distribution for three different radiation fields, representative of a molecular cloud, the diffuse interstellar medium (DIM), and the Orion Bar. We see that the most probable energy increases when the radiation field intensity $G_{0}$ increases, as expected for PAHs whose cooling by IR emission is interrupted more frequently by absorption events. The sharp cut-off at $\sim 1.1 \times 10^{5} \mathrm{~cm}^{-1}$ stems from the Lyman limit of photon energies in neutral regions. We also observe a tail at higher energies that comes from to multiphoton events, i.e., absorption of a second photon while the PAH has not completely cooled off. This tail becomes more significant as absorption events are more frequent, i.e., for large PAHs or intense radiation fields. Conversely, we show in Fig. 4b that the radiation field hardness has little influence on $P(E)$. To estimate the effect of the rotational absorption that we neglected, we included a band centred at $1 \mathrm{~cm}^{-1}$ of width $1 \mathrm{~cm}^{-1}$ and corresponding to $J=150$ $\left(\sigma / N_{\mathrm{C}} \sim 9 \times 10^{-22} \mathrm{~cm}^{2} / \mathrm{C}\right.$, see Sects. 4.2 and 4.3): we found $P(E)$ to be twice as large between 1 and $10 \mathrm{~cm}^{-1}$ and unchanged otherwise. This would change the emission around $1 \mathrm{~cm}$ at very

\footnotetext{
${ }^{3} G_{0}$ scales the radiation field intensity integrated between 6 and $13.6 \mathrm{eV}$. The Mathis radiation field, $G_{0}=1$, corresponds to an intensity of $1.6 \times 10^{-3} \mathrm{erg} / \mathrm{s} / \mathrm{cm}^{2}$.
} 

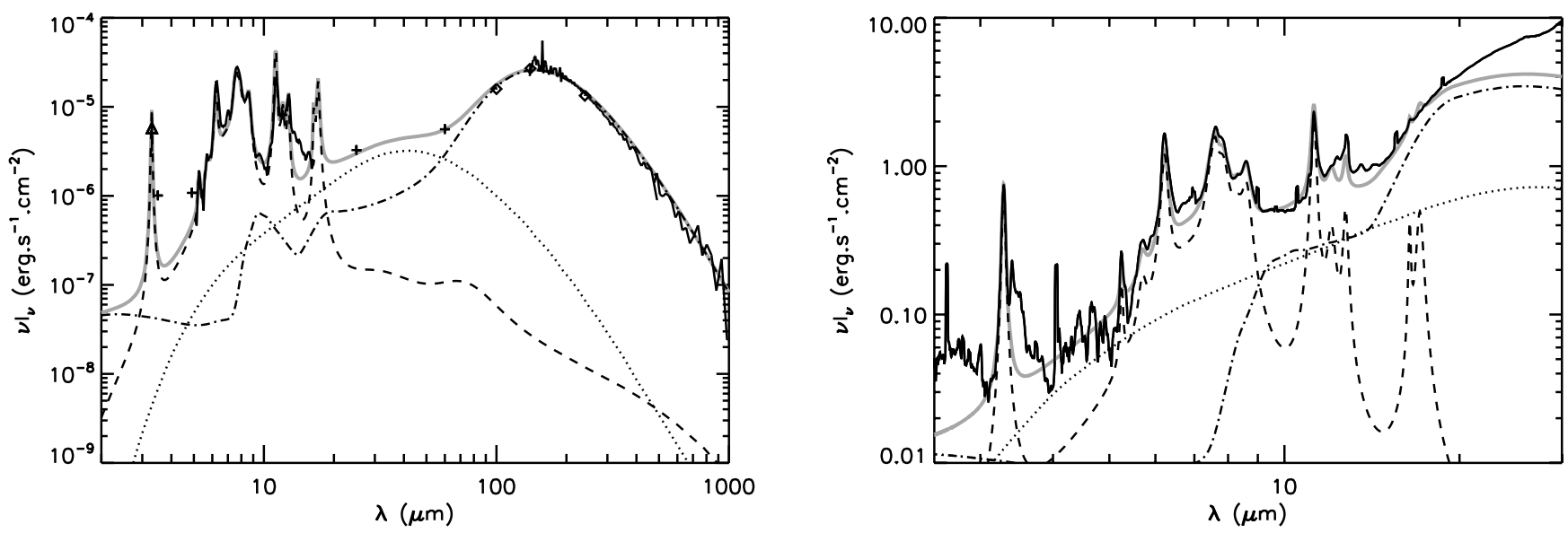

Fig. 5. Left: dust IR emission of the DIM (solid black lines and symbols) with $N_{\mathrm{H}} \sim 10^{20} \mathrm{~cm}^{-2}$ (Boulanger 2000). The model dust emission is overlaid in bold grey. MRN type size distributions are used for all dust populations. The PAH vibrational contribution (dashed line) is from the present model with $N_{\mathrm{C}}=[18,96]$, a 1:3 mixture of neutrals and cations, full hydrogen coverage, and 43 ppm of carbon. The contributions of larger grains are from the model described in Compiègne et al. (2008). The dotted line is the contribution of graphitic very small grains of radii $a=0.9$ to $4 \mathrm{~nm}$ containing $39 \mathrm{ppm}$ of C. The dot-dashed line is the contribution of silicate big grains with $a=0.4$ to $250 \mathrm{~nm}$ using $37 \mathrm{ppm}$ of Si. Right: dust emission from the Orion Bar (the noisy black line is the ISO-SWS spectrum) with $N_{\mathrm{H}} \sim 1.8 \times 10^{21} \mathrm{~cm}^{-2}$. The model dust parameters are as above but for PAHs less abundant (18 ppm of C) and more ionized ( $82 \%$ are cations).

low flux levels. The rotational excitation rates (Sect. 4.2), which depend on the populations of excited vibrational levels, are unaffected by this hypothesis.

Knowing the internal energy distribution of PAHs, we can deduce their IR emission from the upper state $u$ by summing the contributions of all lower vibrational modes $l$ (Draine \& $\mathrm{Li}$ 2001):

$v F_{v}=\frac{2 h v^{4}}{c^{2}} \sigma(v) P(h v)\left(1+\frac{u_{E}}{8 \pi h^{3} v^{3}}\right)$

$$
\text { where } \begin{aligned}
P(h v)= & \sum_{l} P_{l} \sum_{u=0}^{l-1} \frac{g_{u}}{g_{l}} \Delta E_{u} G_{u l}(h v) \\
& +\sum_{l} P_{l}\left(1-\frac{h v}{\Delta E_{l}}\right)
\end{aligned}
$$

is the number of rovibrational photons emitted at energy $h v$. The $G_{u l}$ functions are defined in Draine \& Li (2001). The degeneracies $g_{u}$ and $g_{l}$ are the numbers of energy states in bins $u$ and $l$, respectively (see Appendix B). Figure 5 shows a comparison between mid-IR observations and our model results with a power law size distribution $n_{s}(a) \sim a^{-3.5}$ (as in Mathis et al. (1977) hereafter MRN) that provides a simple representation of the actual $n_{s}(a)$ (Kim et al. 1994). We see that the IR emission from the DIM is explained well with a standard abundance of carbon in PAHs and as large fraction of cations as in Flagey et al. (2006). A similarly good match is obtained for the Orion Bar spectrum with strong PAH depletion probably reflecting an efficient photodestruction in this excited environment. Figure 6a shows the behaviour of the PAH vibrational emission with the radiation field intensity $G_{0}$ : whereas the IR part scales with $G_{0}$, the mm-range $(\lambda>3 \mathrm{~mm})$ does not. The emission in this spectral range is produced mostly by PAHs in low-energy states for which the dominant heating source is absorption of CMB photons, and this is why this part of the spectrum does not vary with $G_{0}$. Finally, we emphasize that at low energies $(\lambda>20 \mu \mathrm{m})$, our model shows the broadband behaviour of the PAH vibrational

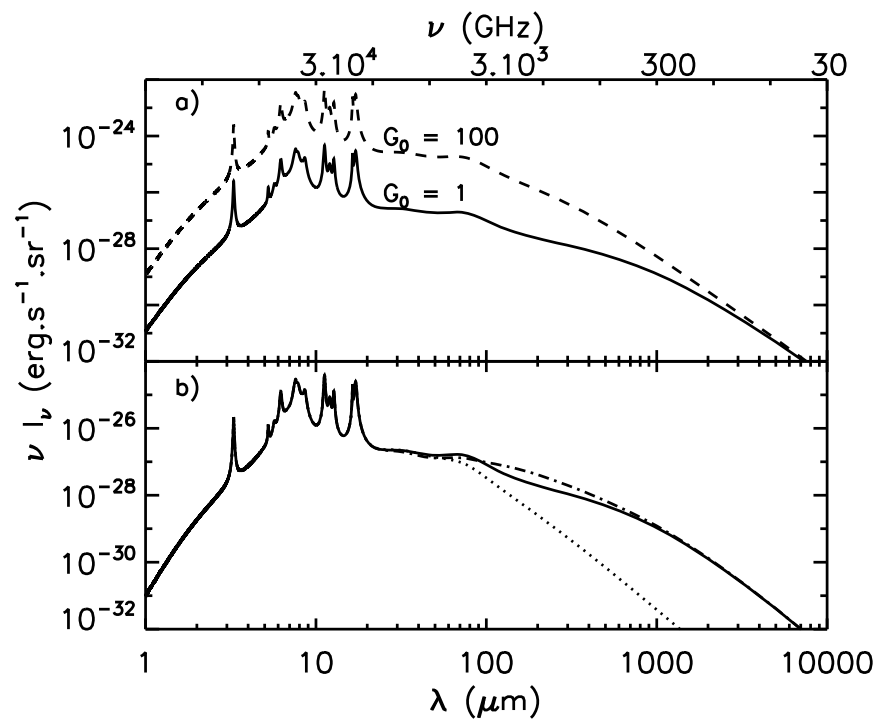

Fig. 6. PAH rovibrational emissivity for a MRN size distribution with $N_{\mathrm{C}}=18-216$. In a) we show the case of radiation field intensities corresponding to $G_{0}=1$ and 100 and in b) we illustrate the effect of IVR breakdown in case 1 (dotted line) and case 2 (dot-dashed line) (see Sect. 3.1).

emission. When a detailed vibrational mode spectrum is used, the PAH emission at $\lambda>20 \mu \mathrm{m}$ is a superposition of numerous narrow bands that may be detectable with some of the instruments onboard Herschel (Mulas et al. 2006).

\subsection{Decoupling of vibrational modes}

Our derivation of $P(E)$ assumes an efficient energy redistribution between vibrational modes (IVR) during the PAH relaxation. The IVR thus involves a coupling between vibrational modes via intramolecular transitions. However, it is known that IVR is no longer efficient when the excitation energy of the molecule 
is below some threshold $E_{\mathrm{dec}}$ (Mulas et al. 2006), and we will then speak of IVR breakdown. When $E<E_{\mathrm{dec}}$, the excitation of energetically accessible vibrational modes is frozen according to microcanonical statistics at energy $E_{\mathrm{dec}}$. The molecule then continues to cool via emission of the excited vibrational modes. To study the influence of decoupling on the long-wavelength emission, we consider two extreme cases for the relaxation at $E<E_{\mathrm{dec}}$ :

- case 1: no IVR, cooling by IR forbidden modes. We assume these modes to be defined like those of Table 1 but with an oscillator strength $10^{4}$ lower and;

- case 2: no IVR, cooling only by the first vibrational mode at $2040 / N_{\mathrm{C}}\left(\mathrm{cm}^{-1}\right)$ as defined in Table 1 ,

and compare them to our model with no decoupling (IVR always fulfilled). We take $E_{\mathrm{dec}}$ to be constant and equal to $500 \mathrm{~cm}^{-1}$, a value relevant for PAHs containing 20 to 30 carbon atoms (Joblin \& Mulas 2008). Actually, $E_{\mathrm{dec}}$ is expected to decrease with molecular size because of the increasing density of states at a given energy (Mulas 1998): the former cases therefore provide an upper limit to the variations in the PAH long-wavelength emissivity. Figure $6 \mathrm{~b}$ shows that, as expected, case 1 provides a lower limit to the long-wavelength emission of PAHs, whereas case 2 is an upper limit. The model assuming IVR is close to case 2. A comparison of our model band fluxes (with IVR) with the Monte-Carlo simulations of Joblin \& Mulas (2008), in the case of the coronene cation $\left(\mathrm{C}_{24} \mathrm{H}_{12}^{+}\right)$, shows good general agreement, especially for the low-energy modes. We conclude that the IVR hypothesis provides an upper limit to the PAH vibrational emission at $\lambda>3 \mathrm{~mm}$ and that the conclusions of the former section hold.

\section{Angular momentum distribution}

Building the angular momentum distribution of interstellar PAHs is driven by photon exchanges and gas-grain interactions (DL98). In the DIM, pervaded by the ISRF, and for a PAH bearing 50 carbon atoms, the mean time between absorptions of visible-UV photons $(\sim 0.2 \mathrm{yr})$ is comparable to the mean time between emissions of rotational photons, as well as to the mean time between PAH-hydrogen collisions (for a gas density of $100 \mathrm{~cm}^{-3}$ and a temperature of $100 \mathrm{~K}$ ). Photons absorbed in the visible-UV have a weak effect on the total angular momentum; indeed, each photon exchanged carries a unit angular momentum, and the numerous ${ }^{4}$ IR photons emitted overwhelm the influence of the photon absorbed. To estimate the angular momentum distribution, we take the following processes into account:

- IR rovibrational photon emission;

- purely rotational photon emission;

- $\mathrm{H}_{2}$ formation;

- collisions with gas (neutral atoms and ions);

- plasma drag;

- photoelectric effect.

All these processes lead to a change in the angular momentum of the molecule, some of them excite the rotation, and others damp it. For the radiative processes we adopt a quantum approach where each rotational state is treated individually and transition rules applied. As discussed by Rouan et al. (1997), the building of the angular momentum distribution, $n(J)$, can be

\footnotetext{
4 Energy conservation implies that 40 IR photons are emitted after each absorption.
}

considered as a stationary random walk in a potential well with a minimum for $J=J_{0}$, for which the rate of $J$-change is zero:

$$
\left(\sum_{i}\left(\tau^{-1} \Delta J\right)_{i}\right)_{J_{0}}=0
$$

with $\Delta J$ the change of $J$ produced by the event number $i$, and $\tau$ the mean time between two events $i$. As in Rouan et al. (1992), we assume an efficient intramolecular vibration to rotation energy transfer (IVRET). We take $n(J)$ to be the same for all vibrational levels, equal to a Maxwell distribution $n(J)=$ $n_{0} J^{2} \exp \left(-J^{2} / J_{0}^{2}\right)$, where $n_{0}$ is a normalization factor. Indeed, Mulas (1998) and Ali-Haïmoud et al. (2009) show that this form of $n(J)$ is a good approximation ${ }^{5}$. In the following, we establish the rate of $J$-change due to the rovibrational emission of isolated PAHs modelled in Sect. 3. We also present the rate of purely rotational photon emission, while the contribution of the gas-grain interactions is described in Appendix D.

\subsection{Rovibrational transitions}

The rovibrational IR emission can be both an exciting and a damping process for the rotation of a PAH. Assuming that the interstellar PAHs are symmetric top molecules, the selection rules for the emission of an IR rovibrational photon are: $\Delta J=0, \pm 1$, and $\Delta K=0, \pm 1$. Transitions corresponding to $\Delta J=+1,-1$, and 0 are called the $P, Q$, and $R$-bands, respectively. As seen in Sect. 2.2, two types of transitions are possible: parallel ones (out-of-plane vibrational motion) with $\Delta K=0$ and perpendicular ones (in-plane vibrational motion) with $\Delta K= \pm 1$. The type of each transition is given in Tables A.1 and 1. For a rovibrational transition, $(v, J, K) \rightarrow(v-1, J+\Delta J, K+\Delta K)$, the rate is proportional to the spontaneous emission coefficient and to the probability for the grain to lose the corresponding transition energy. The spontaneous emission rate is proportional to the $A_{K J}$ factors, which represent the angular part of the transition probability. Formulae for these factors are given in Appendix C. Expressed in terms of cross-section, the transition rate is (with $v_{i}$ and $\Delta v_{i}$ in $\left.\mathrm{cm}^{-1}\right)$ :

$$
W_{i}^{ \pm / 0}=8 \pi c\left(\sigma_{i} \Delta v_{i}\right) \sum_{K=0}^{J}\left(v_{i}^{ \pm / 0}\right)^{2} A_{K J}^{ \pm / 0} P\left(h v_{i}^{ \pm / 0}\right)
$$

where the $W_{i}^{ \pm / 0}$ give the transition rate for $\Delta J=0, \pm 1$, and for the vibrational mode $i$ at a frequency $v_{i}^{ \pm / 0}$. This frequency depends on $v_{i 0}$ (the frequency of the vibrational transition for $J=0$ ) and on $J$ and $K$ (see Appendix C). Since the $J$ - and $K$-terms, in $v_{i}^{ \pm / 0}$, are always much smaller than $v_{i 0}$, we take $P\left(h v_{i}^{ \pm / 0}\right) \simeq P\left(h v_{i 0}\right)$. We note that the in-plane transitions provide higher (by a factor $\sim 2$ ) rates than the out-of-plane ones, as a consequence of the $A_{K J}$ sum values. The total transition rates are obtained by summing over all bands: $W^{ \pm / 0}=\sum_{i} W_{i}^{ \pm / 0}$. Finally, the rate of change in $J$ due to rovibrational transitions is $\left(\tau^{-1} \Delta J\right)_{I R}=W^{+}-W^{-}$. Rotational excitation dominates at low $J$, whereas damping is the dominant process for high $J$-values. For vibrational modes at higher frequency, $W^{+}-W^{-}$is smaller because the rotational terms are smaller in $v_{i}$ (see Appendix C): $\left(\tau^{-1} \Delta J\right)_{\text {IR }}$ is therefore dominated by the contribution of vibrational modes at low-frequencies.

\footnotetext{
5 With this form of $n(J)$ it is possible to define a rotational temperature (see Appendix E).
} 
N. Ysard and L. Verstraete: Long-wavelength emission of interstellar PAHs

Table 2. Physical parameters for the typical interstellar phases considered.

\begin{tabular}{cccccc}
\hline \hline & $\mathrm{MC}^{a}$ & $\mathrm{CNM}^{b}$ & $\mathrm{WNM}^{c}$ & WIM $^{d}$ & Orion Bar \\
\hline$G_{0}$ & 0.01 & 1 & 1 & 1 & 14000 \\
$n_{\mathrm{H}}\left(\mathrm{cm}^{-3}\right)$ & 300 & 30 & 0.4 & 0.1 & $10^{4}$ \\
$T(\mathrm{~K})$ & 20 & 100 & 6000 & 8000 & 400 \\
$n_{\mathrm{e}}\left(\mathrm{cm}^{-3}\right)$ & 0.03 & 0.045 & 0.04 & 0.1 & 3 \\
$n_{\mathrm{H}^{+}} / n_{\mathrm{H}}$ & 0 & $1.2 \times 10^{-3}$ & 0.1 & 0.99 & $10^{-4}$ \\
$n_{\mathrm{C}^{+}} / n_{\mathrm{H}}$ & $10^{-4}$ & $1.3 \times 10^{-4}$ & $1.3 \times 10^{-4}$ & $10^{-3}$ & $2 \times 10^{-4}$ \\
\hline
\end{tabular}

$n_{\mathrm{H}}$ the hydrogen density, $T$ the gas temperature, $n_{\mathrm{e}}$ the electrons density, $n_{\mathrm{H}^{+}}$the proton density, and $n_{\mathrm{C}^{+}}$the density of atomic carbon ions.

${ }^{a}$ Molecular cloud. ${ }^{b}$ Cold neutral medium. ${ }^{c}$ Warm neutral medium.

${ }^{d}$ Warm ionized medium.

\subsection{Rotational emission}

We consider here the spontaneous emission of purely rotational photons. The selection rules for such transitions are $\Delta J=-1$ and $\Delta K=0$ (Townes \& Schawlow 1975). No change in $K$ occurs because, for rotational transitions, the dipole moment of a symmetric top molecule necessarily lies along its symmetry axis. The transition rate is then simply related to the spontaneous emission coefficient $A_{J, J-1}$ :

$$
\begin{aligned}
\left(\tau^{-1} \Delta J\right)_{\text {rot }} & =-A_{J, J-1} \\
A_{J, J-1} & =\frac{512 \pi^{4}}{3 h^{4} c^{3}} B^{3} \mu^{2} J^{3} \frac{(2 J+1)^{2}-(J+2)}{3(2 J+1)^{2}} \mathrm{~s}^{-1}
\end{aligned}
$$

where $\mu$ is the electric dipole moment of the molecule and the factor $A_{K J}$ - has been used (Appendix C). In the high- $J$ limit, $h v \times A_{J, J-1}$ tends to the classical expression of Larmor. Finally, the damping rate due to rotational emission is ${ }^{6}$

$$
\begin{aligned}
\left(\tau^{-1} \Delta J\right)_{\mathrm{rot}}= & -1.8 \times 10^{-14}\left(\frac{N_{\mathrm{C}}}{50}\right)^{-6}\left(\frac{\mu}{1 \mathrm{D}}\right)^{2} \\
& \times J^{3} \frac{(2 J+1)^{2}-(J+2)}{(2 J+1)^{2}} \mathrm{~s}^{-1} .
\end{aligned}
$$

\subsection{Equilibrium angular momentum $J_{0}$}

We estimate damping and excitation rotational rates for the interstellar phases described in Table 2. When solving Eq. (8), we obtain the equilibrium angular momentum $J_{0}$. Figure 7 shows $J_{0}$ for the DIM and the Orion Bar. In all cases, $J_{0}$ increases with $N_{\mathrm{C}}$ because there are more steps in the random walk; indeed, the cross-section and absorption rate scale with $N_{\mathrm{C}}$ (see Fig. 2), so more IR photons are emitted for larger $N_{\mathrm{C}}$. Similarly, $J_{0}$ reaches higher values when the radiation field intensity or the gas density increases (see Fig. 8).

In the DIM, the excitation comes from gas-grain collisions, whereas the damping is dominated by the emission of rotational photons for $N_{\mathrm{C}}<140$ and by IR photon emission for larger PAHs $\left(\left(\tau^{-1} \Delta J\right)_{\text {rot }} \propto N_{\mathrm{C}}^{-5}\right.$, whereas $\left.\left(\tau^{-1} \Delta J\right)_{\mathrm{IR}} \propto N_{\mathrm{C}}\right)$. We note similar $J_{0}$-values for the CNM, WNM and WIM (Fig. 7a) in spite of the large differences in gas density and temperature (see Table 2). In the Orion Bar, because of the intense radiation field, the excitation is driven by the photoelectric effect, whereas the damping is dominated by the emission of IR photons. For the case of MCs (not shown), $J_{0}$ also rises and reaches high values $\left(J_{0}=1300\right.$ for $\left.N_{\mathrm{C}}=200\right)$. Indeed, the damping by IR photon

\footnotetext{
6 In the limit $J \gg 1$, we estimated that the stimulated emission and absorption of CMB photons represent less than $20 \%$ of the spontaneous emission rate.
}

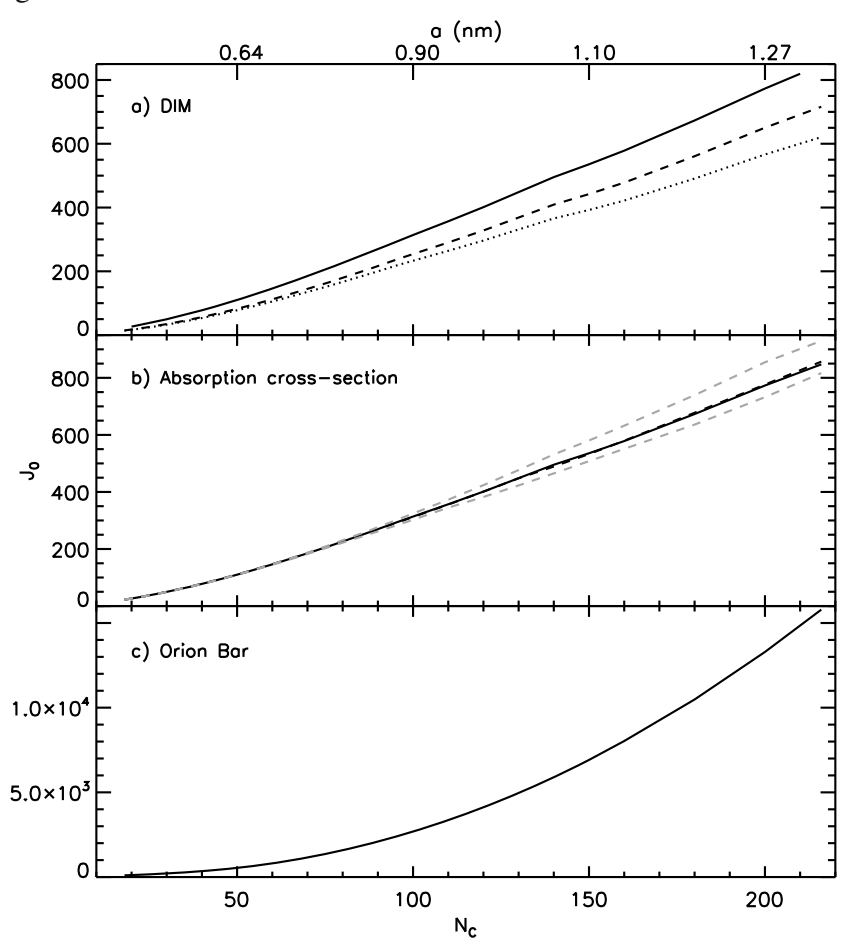

Fig. 7. $J_{0}$ versus $N_{\mathrm{C}}$ for PAH cations. In a) we show the case of the diffuse medium. Solid line shows the CNM, dashed line the WNM and dotted line the WIM. In b) we illustrate the influence of the absorption cross-section in the CNM: cations (solid line), neutrals (dashed line) and extreme behaviours (grey lines) for the first vibrational mode versus $N_{\mathrm{C}}$ (see Fig. 1) are shown. In c), we present the case of the Orion Bar.

emission is no longer efficient $\left(G_{0} \sim 0.01\right)$, while the gas-grain rates are strong because of higher density. We show in Fig. $7 \mathrm{~b}$ the influence of different choices of the vibrational mode spectrum (or cross-section) on $J_{0}$ : changes are below $20 \%$ and affect only the large sizes $\left(N_{\mathrm{C}}>100\right)$, which make a minor contribution to the rotational emissivity (see Sect. 5). We found comparable variations of $J_{0}$ for the vibrational relaxation (IVR) cases 1 and 2, discussed in Sect. 3.1.

The processes contributing to the rotation of PAHs depend on the intensity of the radiation field $\left(G_{0}\right)$ and on the gas density $\left(n_{\mathrm{H}}\right)$. We now examine the influence of these parameters on $J_{0}$. Other important quantities (gas temperature; electron, proton, and $\mathrm{C}^{+}$abundances) are obtained at thermal equilibrium with CLOUDY (Ferland et al. 1998) ${ }^{7}$. To study the influence of $G_{0}$, we took the radiation field to be a blackbody of effective temperature $22000 \mathrm{~K}$ and scaled it in order to vary $G_{0}$ between $10^{-2}$ and $10^{5}$. The shape of the radiation field is then always the same ${ }^{8}$. Figure 8 a shows $J_{0}$ as a function of $G_{0}$ for different PAH sizes. As discussed before, $J_{0}$ increases with $G_{0}$ because the excitation rate from IR emission (which scales with $G_{0}$ ) is dominant. This rise becomes steeper for larger PAHs because the frequency of the first vibrational mode decreases (as $N_{\mathrm{C}}^{-1}$ ) and requires lower values of $G_{0}$ to be excited (the maximum of $P(E)$ moves to higher energies as $G_{0}$ increases, see Fig. 4c). However, we note that $J_{0}$ is affected little by variations of $G_{0}$ over the range $0.01-100$. The result of varying $n_{\mathrm{H}}$ is shown in

\footnotetext{
7 We assumed 130 (320) ppm of carbon (oxygen) to be in the gas phase. Parameters were taken from the optically thin zone of isochoric simulations with CLOUDY.

${ }^{8}$ We also varied the shape of the blackbody keeping $G_{0}$ constant (as well as $n_{\mathrm{H}}$ ). Similar $J_{0}$ values were found for $T_{\text {eff }}$ between $10^{4}$ and $5 \times$ $10^{4} \mathrm{~K}$.
} 

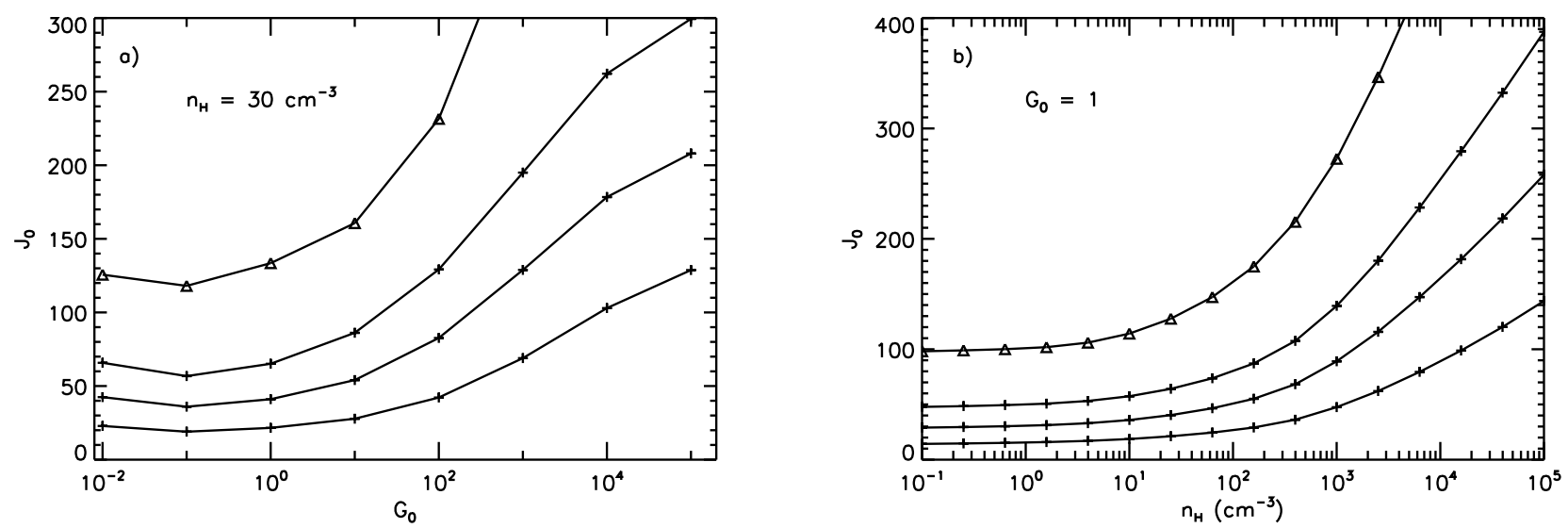

Fig. 8. a) Effect of varying $G_{0}$ on $J_{0}$ for PAH cations with $N_{\mathrm{C}}=20,30,40$, and 100 (from bottom to top). The values for $N_{\mathrm{C}}=100$ (triangles) have been divided by 2 . The gas density is $30 \mathrm{~cm}^{-3}$ and the other physical parameters for the gas are determined using CLOUDY (Ferland et al. 1998). b) Same as a) but for varying $n_{\mathrm{H}}$ at $G_{0}=1$. In both cases the radiation field is a blackbody with $T_{\text {eff }}=22000 \mathrm{~K}$ and the gas parameters have been obtained with CLOUDY at thermal equilibrium (see text).

Fig. 8b. In all cases, the incident radiation field is a blackbody with $T_{\text {eff }}=22000 \mathrm{~K}$ and $G_{0}=1$. The gas-grain processes become dominant for $n_{\mathrm{H}}>30 \mathrm{~cm}^{-3}$ and $N_{\mathrm{C}}>50$. At lower gas densities, radiative processes prevail (excitation by IR emission and damping by rotational emission), and $J_{0}$ does not change. We therefore expect the rotational excitation of small PAHs to hardly be variable in the DIM as found by Davies et al. (2006).

\subsection{Modelling the rotational excitation by IR emission}

Following the absorption of a stellar visible-UV photon, the rovibrational cascade of PAHs is a complex process that involves many states. Molecular, state-to-state models require a detailed database, use Monte-Carlo simulations (see Mulas et al. 2006, for references), and so far do not include the gas-grain interactions that are important. Given our incomplete knowledge of interstellar PAHs and the fact that their rotational emission may so far be seen in broadband data, former models of spinning dust (DL98 and Ali-Haïmoud et al. 2009) made simplifying assumptions to describe radiative processes and performed a classical treatment of the gas-grain interactions ${ }^{9}$. We assess here the impact of these assumptions on the rotational emissivity of PAHs.

First, the internal energy distribution of PAHs was derived in the thermal approximation. As discussed in Sect. 4, this is a questionable assumption for describing the long-wavelength emission of PAHs and the change in angular momentum it induces. Moreover, the excitation rate by IR emission (the recoil due to emission of individual photons), which is a purely quantum effect, has been described as a random walk of the angular momentum starting with a non-rotating grain ${ }^{10}$. Finally, an efficient vibrational redistribution (IVR, see Sect. 3.1) was assumed throughout the energy cascade following a photon absorption. In this work, we improve on the first aspect by deriving the internal energy distribution of isolated PAHs using a microcanonical formalism and including the rotational density of states. Next, we follow a quantum approach to treat the rovibrational emission where the recoil due to photon emission and selection rules are

\footnotetext{
9 These models can also be applied to describe the rotational emission of other grain types.

10 Conversely, the damping part is correctly described by a classical model when $J \gg 1$ (Ali-Haïmoud et al. 2009).
}

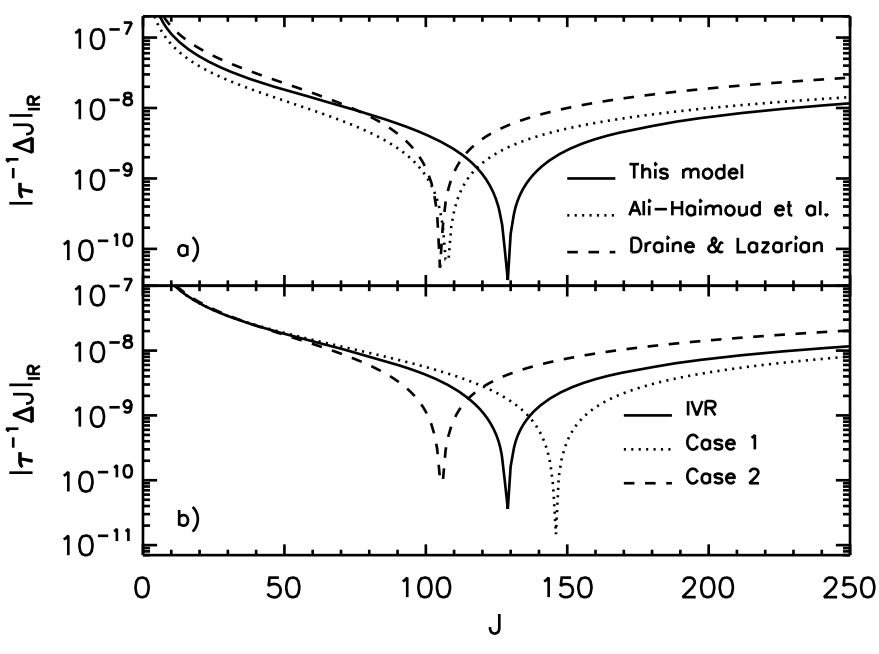

Fig. 9. Rate of $J$-change due to rovibrational emission for a PAH with $N_{\mathrm{C}}=24$ and heated by the ISRF. a) Comparison with former models: our rate (solid line), the rate of DL98 corrected as in Ali-Haïmoud et al. (2009) (dashed line) and the rate of Ali-Haïmoud et al. (2009) (dotted line). b) Effect of IVR breakdown, case 1 and 2 (see Sect. 3.1).

naturally included. In Fig. 9a, we compare the absolute value of our rate of angular momentum change by rovibrational IR emission $\left|\left(\tau^{-1} \Delta J\right)_{\mathrm{IR}}\right|=\left|W^{+}-W^{-}\right|$(see Sect. 4.1) to former works. For a PAH containing 24 carbon atoms, all rates decrease with the angular momentum and cross zero for $J$ between 100 and 150 (singular points in our logarithmic representation). The main difference with previous models is that the excitation-to-damping transition (the zero value) occurs at higher $J$-values in our case. This discrepancy diminishes with increasing sizes, and identical IR rates are found for species with more than $100 \mathrm{C}$ atoms.

We show in Fig. 9b the effect of relaxation schemes other than IVR. Between extreme cases 1 and 2 (see Sect. 3.1), the damping IR rate is multiplied by a factor 2 , which leads to a balance at a lower $J$-value. Variations in $\left(\tau^{-1} \Delta J\right)_{\mathrm{IR}}$ are in fact quite similar to those induced by the other approximations discussed above. All these variations affect the rotational emissivity of PAHs, around $30 \mathrm{GHz}$, by at most $15 \%$ (in the CNM and 

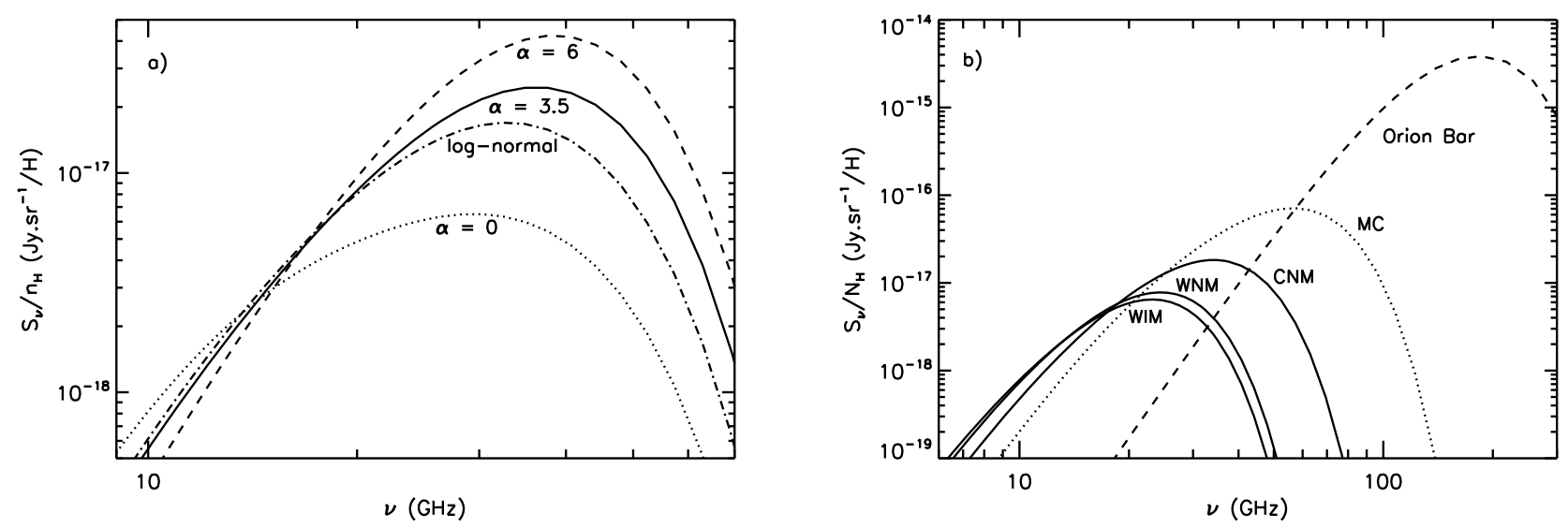

Fig. 10. Rotational emissivity of PAH cations. We take $430 \mathrm{ppm}$ of carbon in PAHs, $N_{\mathrm{C}}=24-216$ and assume $m=0.4$ Debye. Panel a): effect of changing the fraction of large PAHs (decreasing with $\alpha=0$ to 6). The case of a log-normal distribution centered around $N_{\mathrm{C}}=44$ and width 0.4 is also shown. Panel b): rotational emission spectrum in different environments with a power law size distribution and $\alpha=3.5$.

including the gas-grain interactions $)^{11}$. We conclude that, in spite of the assumptions made, former spinning dust models (which are fast computationally) provide a sufficiently accurate rotational emissivity.

\section{Spinning dust emission}

The power emitted by a PAH containing $N_{\mathrm{C}}$ carbon atoms in a rotational transition from state $J$ to $J-1$ is equal to

$P(J)=A_{J, J-1} \times 2 B J$,

when taking the spontaneous emission rate $A_{J, J-1}$ from Eq. (10) and with the transition energy $h v=2 B J(\Delta K=0$ for rotational transitions). With $B \propto N_{\mathrm{C}}^{-2}$ and $\mu^{2} \propto N_{\mathrm{C}}$, we find that $P(J) \propto N_{\mathrm{C}}^{-7}$ : small PAHs will therefore have a dominant contribution to the rotational emission. For instance, the peak value of the rotational emissivity of a PAH with $N_{\mathrm{C}}=96$ is 10 times lower than for a species with $N_{\mathrm{C}}=24$ (see also Ali-Haïmoud et al. 2009). With $n_{s}$, the size distribution of PAHs (the number of PAHs of a given size $a$ or $N_{\mathrm{C}}$ per proton), and the angular momentum distribution $n\left(N_{\mathrm{C}}, J\right)$, we get

$S_{v}=\frac{N_{\mathrm{H}}}{4 \pi} \int_{N_{\min }}^{N_{\max }} A_{J \rightarrow J-1} n\left(N_{\mathrm{C}}, J\right) \frac{2 B J}{2 B c} n_{s}\left(N_{\mathrm{C}}\right) \mathrm{d} N_{\mathrm{C}}$.

Since we are interested in a broadband spectrum, we take the rotational bandwidth to be $2 B c$. As discussed before, we assume that $n\left(N_{\mathrm{C}}, J\right)$ is described correctly by a Maxwell distribution. Given the relationship $J_{0}\left(N_{\mathrm{C}}\right)$ (Fig. 7) and the size distribution, the emission of spinning PAHs can be calculated from Eq. (14). It scales with $m^{2} \times S_{\text {PAH }}$. We illustrate the relative significance of small species in Fig. 10a by changing the index of the power law size distribution, $n_{s}(a) \sim a^{-\alpha}$. Defining small PAHs by $N_{\mathrm{C}} \lesssim$ 100 , they represent $50 \%(97 \%)$ of the total abundance for $\alpha=0$ (6). As expected, the rotational emissivity for $\alpha=6$ is highest. It is also blueshifted by $5 \mathrm{GHz}$ and broadened by about the same amount with respect to the $\alpha=0$ case. Figure $10 \mathrm{~b}$ shows the case of different interstellar environments and illustrate the influence

11 A correction of the same order was found by Ali-Haïmoud et al. (2009), who used the Fokker-Planck equation to derive $n(J)$ instead of a Maxwell distribution. of the gas-grain processes, which become strong in the CNM (while subdominant in the WIM/WNM).

We emphasize here that the level, peak position and width of the rotational emission spectrum depend on (a) the fraction of small PAHs $\left(N_{\mathrm{C}} \leq 100\right)$, (b) $m$ the scaling factor for the dipole moment, and (c) the physical parameters of the gas along the line of sight (temperature, density, and ionization). Microwave observations alone cannot constrain all these unknowns. A quantitative description of the spinning dust emission will require nearIR data (to constrain the fraction of small PAHs) and radio maps (21 cm and continuum) to derive the physical state of the gas.

\section{The case of the molecular cloud G159.6-18.5}

We present here a comparison of our model results with observations of the anomalous microwave foreground and IR emission. We show how these data can be explained with a coherent description of the IR and rotational emission of PAHs, and also discuss how the size distribution of PAHs can be constrained.

The COSMOSOMAS experiment has delivered maps of large sky fractions with an angular resolution of 1 degree in four frequency bands: $10.9,12.7,14.7$, and $16.3 \mathrm{GHz}$. In this survey, Watson et al. (2005) studied the region G159.6-18.5 located in the Perseus molecular complex. Figure 11 shows the spectrum of this region from 3 to to $4000 \mathrm{GHz}$ (COSMOSOMAS, WMAP, and DIRBE data). From mid to far-IR, the Perseus molecular complex is dominated by a molecular ring (G159.6-18.5) surrounding an HII region. Unfortunately, the COSMOSOMAS resolution does not allow these two phases to be separated. The molecular ring is centred on the star HD 278942 (Andersson et al. 2000; Ridge et al. 2006). Its stellar wind is responsible for the HII region dug into the parent molecular cloud. To describe the emission spectrum of G159-18.5, we assume it includes an MC component for the ring and a WIM component for the HII region. We discuss below the physical parameters of both phases.

According to Ridge et al. (2006), HD278942 is a B0 V star: its radiation field is modelled by a blackbody with $T_{\text {eff }} \sim$ $30000 \mathrm{~K}$, scaled to get $G_{0} \sim 1.6$ at the radius of the ring $\left(G_{0}\right.$ is obtained from IRAS data). The radius of the HII region is approximately $2.75 \mathrm{pc}$ (Andersson et al. 2000) and implies a WIM density $n_{\mathrm{H}} \sim 1 \mathrm{~cm}^{-3}$ (Ridge et al. 2006). We obtained the WIM temperature from CLOUDY: $7500 \mathrm{~K}$. Andersson et al. (2000) 


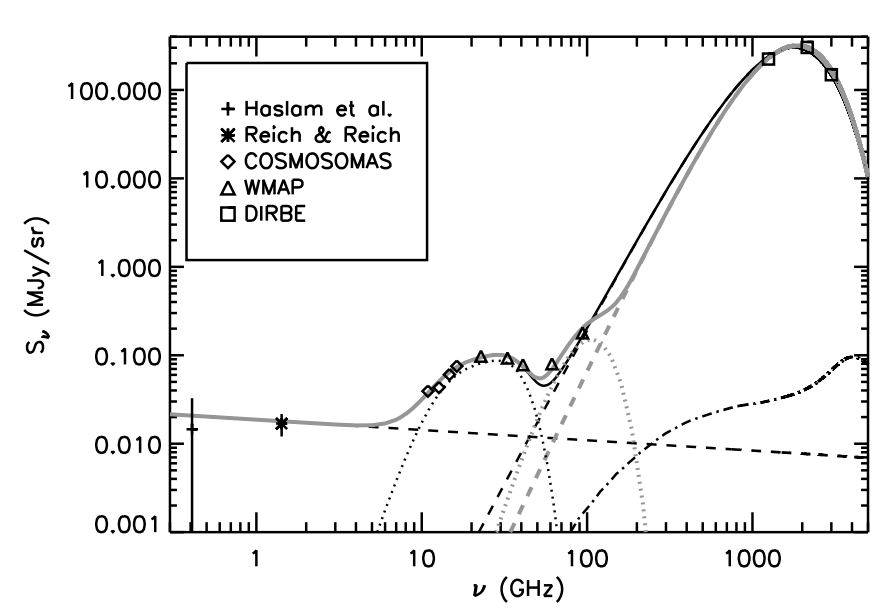

Fig. 11. Comparison to observations (symbols) of the Perseus molecular cloud G159.6-18.5. The dashed lines show the free-free (low frequency) and big grains (high-frequency) emissions. The PAH rovibrational emission is represented by the dot-dashed line. Dotted lines show the PAH rotational emission. The black model is model A, and the grey model is model B. See text for details.

estimated the density and temperature of the gas in the molecular ring to be $n_{\mathrm{H}} \sim 560 \mathrm{~cm}^{-3}$ and $T=(20 \pm 10) \mathrm{K}$. For the WIM and MC phases, we derived the abundances of electrons, protons and $\mathrm{C}^{+}$, and the gas temperature with CLOUDY. We took the total column density (MC+WIM) to be $N_{\mathrm{H}}=1.3 \times 10^{22} \mathrm{~cm}^{-2}$ (Watson et al. 2005). From $12 \mu \mathrm{m}$ IRAS data and our PAH emission model, we estimated the total PAH abundance to represent $(240 \pm 40)$ ppm of carbon.

Following Watson et al. (2005), we took the thermal dust emission to be a grey body at $19 \mathrm{~K}$ with an emissivity index $\beta=1.55$. Our best-fit parameters for the spinning PAH contribution (assuming a size distribution $n_{s}(a) \sim a^{-3.5}$ ) are then (model $A) N_{\mathrm{C}}=50-216$ and $N_{\mathrm{H}}=4.6 \times 10^{21} \mathrm{~cm}^{-2}$ for the MC, $N_{\mathrm{C}}=24-216$ and $N_{\mathrm{H}}=8.1 \times 10^{21} \mathrm{~cm}^{-2}$ for the WIM. In both media, we took $m=0.6 \mathrm{D}$ (Eq. (4)). Larger PAHs are required in the $\mathrm{MC}$, possibly as a consequence of grain-grain coagulation. However, the results of Dupac et al. (2003) indicate that emission of dust around $20 \mathrm{~K}$ requires a higher emissivity index, close to 2 . When adopting $\beta=2$ and a dust temperature of $18 \mathrm{~K}($ model $B)$, we find an equally good fit of this region in the far-IR (Fig. 11). In this case, the flux around $100 \mathrm{GHz}$ is not explained. We speculate here that it may come from an additional and less abundant population of small PAHs in the MC component with: $N_{\mathrm{C}}=24-50, m=0.1 \mathrm{D}$ and $N_{\mathrm{H}}=1.25 \times 10^{21} \mathrm{~cm}^{-2}$ (for the larger PAHs in the MC phase, $N_{\mathrm{H}}=4.25 \times 10^{21} \mathrm{~cm}^{-2}$ ). The corresponding spinning emission is shown in Fig. 11 (model $B)$. The PAH size distribution would thus be bimodal, as already suggested by Le Page et al. (2003). As can be seen in Fig. 11, both models provide a good fit to the data. Discrimiting tests of these scenarios will be soon possible with the Planck polarized data. Small grains or PAHs are expected to be poorly aligned (Lazarian \& Draine 2000; Martin 2007), whereas big grains are fairly well-aligned with a polarization fraction of 5 to $10 \%$.

\section{Summary}

The Planck and Herschel data will soon reveal the emission of interstellar dust at long wavelengths. Thanks to their small size, interstellar PAHs spend most of their time at low internal energy and can spin at frequencies of a few tens of GHz. The emission of PAHs is therefore expected to make a significant contribution at long wavelength. Recent observations have shown the existence of a 10-100 GHz emission component (the anomalous foreground), related to the smallest dust grains. As suggested by DL98, the anomalous foreground may trace the emission of spinning PAHs.

In this work, we built the first model that coherently describes the emission of interstellar PAHs from the near-IR to the centimetric range and focused on the long wavelength part of this emission. To do so, we derived the internal energy of isolated PAHs down to low energies. We included low-frequency vibrational bands $(\lambda>20 \mu \mathrm{m})$, through which PAHs cool at intermediate to low energies. They are important for their rotational emission. In the cooling cascade that follows the absorption of a stellar photon by a PAH, we treated rovibrational transitions in a quantum approach and examined the possibility that the hypothesis of vibrational redistribution (IVR) is not always fulfilled. Purely quantum effects (recoil due to photon emission; transitions that do not change the angular momentum or $Q$-bands) are thus naturally included in our description of the rovibrational cascade. We obtained the rotational emission of PAHs from a random-walk formalism, including all processes participating in excitation and damping, namely, rovibrational and pure rotational transitions and gas-grain interactions.

We have shown that the rovibrational emission of PAHs above $\sim 3 \mathrm{~mm}$ does not depend on the intensity of the radiation field (represented by $G_{0}$ ), unlike the mid-IR part of the spectrum that scales linearly with it. In the diffuse interstellar medium, PAHs may contribute up to $10 \%$ of the dust emission around $100 \mathrm{GHz}$. We also found the rotational emissivity of PAHs is dominated by small species (bearing less than $100 \mathrm{C}$ atoms) and is hardly sensitive to $G_{0}$ over the range $0.1-100$. Using plausible PAH properties, our model can explain both the IR and microwave emissions of a molecular cloud in the Perseus arm where the anomalous foreground is conspicuous. The level, peak position and width of the rotational emission spectrum depend on the fraction of small PAHs, the dipole moment distribution ( $m$-factor), and the physical parameters of the gas phases present along the line of sight. A quantitative description of the emission of spinning PAHs will therefore involve observations at IR and radio wavelengths $(21 \mathrm{~cm}$ and continuum). Comparing the rotational excitation rate obtained from our quantum treatment of the rovibrational cascade to former works, we showed that the classical approximation used so far has little effect on the rotational emissivity (the peak value varies by at most $15 \%$ ). Similarly, departures from the IVR hypothesis lead to similar emissivity changes. We therefore conclude that a classical description of rovibrational transitions and the IVR hypothesis are good approximations for describing the rotational emission of PAHs.

Our results on the influence of the radiation field intensity led to a specific prediction that can be tested observationally. If the anomalous microwave foreground comes from spinning PAHs, it is expected to be correlated with the dust emission in the $12 \mu \mathrm{m}$ IRAS band, mostly carried by PAHs. In regions where $G_{0}$ varies significantly, this correlation should improve when the $12 \mu \mathrm{m}$ flux is divided by $G_{0}$, indeed, the IR emission of PAHs scales with $G_{0}$, whereas their rotational emission is independent of $G_{0}$. This prediction was tested in a companion paper with WMAP and IRAS data (Ysard et al. 2010).

Acknowledgements. We thank an anonymous referee whose comments helped us to significantly improve the content of this paper. We gratefully acknowledge stimulating discussions with B. Draine, C. Joblin, E. Dartois, T. Pino, and O. Pirali. We are grateful to $\mathrm{M}$. Compiègne for his help in the dust SED modelling. 
Table A.1. Mid-IR bands of interstellar PAHs adopted in this work for cations and neutrals.

\begin{tabular}{cccccc}
\hline \hline $\begin{array}{c}\lambda_{i} \\
(\mu \mathrm{m})\end{array}$ & $\begin{array}{c}v_{i} \\
\left(\mathrm{~cm}^{-1}\right)\end{array}$ & $\begin{array}{c}\Delta v_{i} \\
\left(\mathrm{~cm}^{-1}\right)\end{array}$ & $\begin{array}{c}\sigma_{i} / N_{\mathrm{H}} \\
\left(10^{-20} \mathrm{~cm}^{2}\right) \\
\text { cations }\end{array}$ & $\begin{array}{c}\sigma_{i} / N_{\mathrm{H}} \\
\left(10^{-20} \mathrm{~cm}^{2}\right) \\
\text { neutrals }\end{array}$ & Type $^{a}$ \\
\hline 3.3 & 3040 & 39 & 2.44 & 10.8 & ip \\
5.2 & 1905 & 23 & 0.58 & 0.58 & op \\
5.7 & 1754 & 60 & 0.49 & 0.49 & op \\
8.3 & 1205 & 63 & 1.74 & 1.74 & ip \\
8.6 & 1162 & 47 & 5.34 & 0.51 & ip \\
11.3 & 890 & 18 & 17.3 & 18.3 & op \\
12.0 & 830 & 30 & 3.17 & 3.17 & op \\
12.7 & 785 & 16 & 5.06 & 4.06 & op \\
\hline$\lambda_{i}$ & $v_{i}$ & $\Delta v_{i}$ & $\sigma_{i} / N_{\mathrm{C}}$ & $\sigma_{i} / N_{\mathrm{C}}$ & Type \\
$(\mu \mathrm{m})$ & $\left(\mathrm{cm}^{-1}\right)$ & $\left(\mathrm{cm}^{-1}\right)$ & $\left(10^{-20} \mathrm{~cm}{ }^{2}\right)$ & $\left.\left(10^{-20} \mathrm{~cm}\right)^{2}\right)$ & \\
& & & cations & neutrals & \\
\hline 6.2 & 1609 & 44 & 2.48 & 0.52 & ip \\
6.9 & 1450 & 300 & 0.40 & 0.40 & ip \\
7.5 & 1328 & 70 & 2.70 & 0.12 & ip \\
7.6 & 1315 & 25 & 1.38 & 0.06 & ip \\
7.8 & 1275 & 70 & 2.70 & 0.12 & ip \\
16.4 & 609 & 6 & 1.83 & 1.83 & ip \\
17.1 & 585 & 17 & 2.48 & 2.48 & ip \\
\hline
\end{tabular}

In-plane (ip) or out-of-plane (op) character of the bands (see Sect. 2.2).

\section{Appendix A: Mid-IR absorption cross-section}

In Table A.1 we give the parameters defining the mid-IR vibrational bands considered in this work. In all cases we assume their profile to have a Drude shape. The bands at 3.3, 6.2, 7.7, 8.6, 11.3 , and $12.7 \mu \mathrm{m}$ are defined as follows. For the PAH cations, we start from the integrated cross-sections, $\sigma \Delta v$, of Pech et al. (2002) that have been derived from laboratory data. The corresponding band profiles, however, do not provide a detailed match of observations. We therefore use band positions and widths as deduced from fits of ISO-SWS spectra of a number of interstellar regions (Verstraete et al. 2001). As indicated by these observations and others (Peeters et al. 2002), we include a broad band at $6.9 \mu \mathrm{m}$ and split the $7.7 \mu \mathrm{m}$ into three sub-bands at 7.5, 7.6 , and $7.8 \mu \mathrm{m}$, where we use the observed $\sigma \Delta v$ of each subband as weights in defining their integrated cross-sections. In addition, we introduce a band at $8.3 \mu \mathrm{m}$ to fill the gap between the 7.8 and $8.6 \mu \mathrm{m}$ bands, and multiply the $8.6 \mu \mathrm{m}$ band by a factor 3 to match observations. For neutral PAHs, we use the laboratory integrated cross-section of Joblin et al. (1995), and assume the same band profiles as for the cations. Furthermore, spectroscopic data (ISO-SWS, Spitzer -IRS and UKIRT) reveal other bands at 5.25 and $5.75 \mu \mathrm{m}$, which have been ascribed to combinations of PAH vibrational modes involving the $11.3 \mu \mathrm{m}$ band and IR-forbidden modes at 9.8 and $11.7 \mu \mathrm{m}$, respectively (Roche et al. 1996; Tripathi et al. 2001). For the $5.25 \mu \mathrm{m}$, we use the width and intensity ratio to the $11.3 \mu \mathrm{m}$ band given in Roche et al. (1996). The $5.75 \mu \mathrm{m}$ band has been derived from the observed spectrum of the Orion Bar (Verstraete et al. 2001). We also add the $17.1 \mu \mathrm{m}$ band recently seen in Spitzer data (Smith et al. 2004; Werner et al. 2004) and recognized as arising from PAHs (Peeters et al. 2004b; Smith et al. 2007).

\section{Appendix B: Vibrational modes and density of states of interstellar PAHs}

\section{B.1. Vibrational modes}

Symmetric top ( $D_{6 \mathrm{~h}}$ symmetry) type PAHs, with $N_{\mathrm{C}}$ carbon atoms and $N_{\mathrm{H}}=\sqrt{6 N_{\mathrm{C}}}$ hydrogen atoms, have $3\left(N_{\mathrm{C}}+N_{\mathrm{H}}-2\right)$ vibrational modes that can be divided into the following types: $\left(N_{\mathrm{C}}-2\right)$ out-of-plane (op) CC modes, $2\left(N_{\mathrm{C}}-2\right)$ in-plane (ip) CC modes, $N_{\mathrm{H}}$ out-of-plane $\mathrm{CH}$ bending modes, $N_{\mathrm{H}}$ in-plane $\mathrm{CH}$ bending modes, and $N_{\mathrm{H}} \mathrm{CH}$ stretching (st) modes. Following Draine \& Li (2001), we approximate the mode spectrum of each type of vibration with a two-dimensional Debye model of maximum energy $k \Theta$, where $\Theta$ is the Debye temperature. We derive the mode spectra from the following expressions:

- for the CC modes:

$$
\hbar \omega_{i}=k \Theta_{t} \sqrt{\frac{i-\delta_{i}^{t}}{N_{t}}} \text { for } i=1, N_{t}
$$

where $t=$ op, ip is the type of mode and $N_{t}$ is the number of $\mathrm{CC}$ modes of a given type with $\Theta_{\mathrm{op}}=876 \mathrm{~K}$ and $\Theta_{\text {ip }}=$ $2318 \mathrm{~K}$ with:

$$
\begin{aligned}
& \delta_{i}^{\mathrm{op}}=3 / 2 \text { for } i=2,3 \text { and } \delta_{i}^{\mathrm{ip}}=1 \text { for } i=2,3 \\
& =3 / 4 \text { otherwise } \quad=1 / 2 \text { otherwise }
\end{aligned}
$$

- for the $\mathrm{CH}$ modes:

$$
\hbar \omega_{i}=k \Theta_{t} \sqrt{\frac{3 i}{2 N_{t}}} \text { for } i=1, N_{t}
$$

where $t=\mathrm{op}$, ip, st, and $N_{t}$ is the number of $\mathrm{CH}$ modes of a given type with $\Theta_{\text {op }}=1281 \mathrm{~K}, \Theta_{\text {ip }}=1672 \mathrm{~K}$ and $\Theta_{\text {st }}=$ $4375 \mathrm{~K}$.

To this mode spectrum, we add the mode at lowest energy as described in Sect. 2.1. Figure B.1 shows that our Debye mode spectra agree with that derived from the Malloci et al. (2007) database.

\section{B.2. Density of states and degeneracies}

We estimate the PAH density of harmonic vibrational states from direct counts, a mode-to-mode convolution method proposed by Beyer \& Swinehart (1973). We start from the rotational density of states, classically given by $\rho_{\text {rot }}(E)=\sqrt{8 E} / B^{3 / 2}$ with $E$ and $B$ in $\mathrm{cm}^{-1}$ and for a symmetric top (Baer \& Hase 1996). We then obtain the rovibrational density of states $\rho(E)$ with the convolution method. The molecule's zero-point energy has been chosen as the zero of the energy scale, and the calculation was made for bins with finite width of $1 \mathrm{~cm}^{-1}$. To calculate the internal energy distribution of PAHs $P(E)$, we grouped this very large number of points into broader energy bins $\left[E_{i}^{\mathrm{min}}, E_{i}^{\mathrm{max}}\right]$ with $i=1$ to 500 . Each energy bin $i$ thus contains many states and its degeneracy, $g_{i}$, is estimated as (with $g_{1}=1$ )

$g_{i}=\int_{E_{i}^{\min }}^{E_{i}^{\max }} \rho(E) \mathrm{d} E \quad(i>1)$.

\section{Appendix C: Lines intensities in the rotational bands}

In the case of symmetric top molecules, we give below the Hönl-London factors for the angular part of rovibrational transition rates, as well as the corresponding transition energies (Herzberg 1968ab). The frequency of the vibrational mode $i$ is noted $v_{i 0}$. 


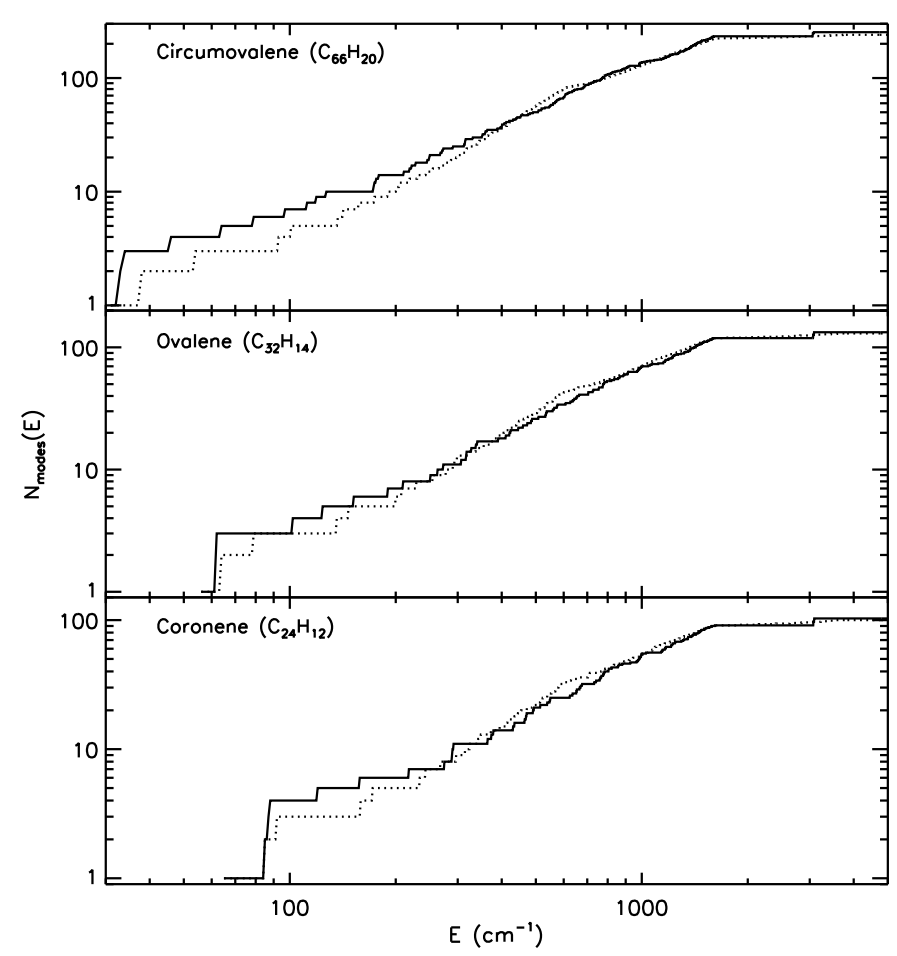

Fig. B.1. Cumulative distribution of vibrational modes for coronene $\left(\mathrm{C}_{24} \mathrm{H}_{12}\right)$, ovalene $\left(\mathrm{C}_{32} \mathrm{H}_{14}\right)$ and circumovalene $\left(\mathrm{C}_{66} \mathrm{H}_{20}\right)$, from the Malloci et al. (2007) database (solid lines) and with our Debye model (dotted lines).

- for $\Delta J=0, \pm 1$ and $\Delta K=0$ (parallel transitions or out of plane transitions):

$$
\begin{array}{ll}
A_{K J^{+}}=\frac{2\left((J+1)^{2}-K^{2}\right)}{(J+1)(2 J+1)^{2}} & \text { and } \quad v_{i}^{+}=v_{i 0}-2 B(J+1) \\
A_{K J^{-}}=\frac{2\left(J^{2}-K^{2}\right)}{J(2 J+1)^{2}} & \text { and } \quad v_{i}^{-}=v_{i 0}+2 B J \\
A_{K J^{0}}=\frac{2 K^{2}}{J(J+1)(2 J+1)} & \text { and } \quad v_{i}^{0}=v_{i 0}
\end{array}
$$

- for $\Delta J=0, \pm 1$ and $\Delta K= \pm 1$ (perpendicular or in plane transitions):

$$
\begin{aligned}
& \left\{\begin{array}{l}
A_{K J^{+}}=\frac{(J+2 \pm K)(J+1 \pm K)}{2(J+1)(2 J+1)^{2}} \\
v_{i}^{+}=v_{i 0}-2 B(J+1)+(B-C)(1 \pm 2 K)
\end{array}\right. \\
& \left\{\begin{array}{l}
A_{K J^{-}}=\frac{(J-1 \mp K)(J \mp K)}{2 J(2 J+1)^{2}} \\
v_{i}^{-}=v_{i 0}+2 B J+(B-C)(1 \pm 2 K)
\end{array}\right. \\
& \left\{\begin{array}{l}
A_{K J^{0}}=\frac{(J+1 \pm K)(J \mp K)}{2 J(J+1)(2 J+1)} \\
v_{i}^{0}=v_{i 0}+(B-C)(1 \pm 2 K)
\end{array}\right.
\end{aligned}
$$

\section{Appendix D: Rate of angular momentum change for gas-grain interactions}

We describe below the gas-grain interactions considered in our model of PAH rotation and the rate of change of $J$ they induce, $\left(\tau^{-1} \Delta J\right)$

\section{D.1. Collisions with gas atoms and plasma drag}

For the collisions with gas neutrals and ions, as well as the plasma drag, we apply the results of DL98 to planar PAHs. In the case where $J \gg 1$, we use the correspondence principle to write $\hbar J=I_{\mathrm{c}} \omega$ and obtain the following rates:

$$
\left(\tau^{-1} \Delta J\right)=-5.2 \times 10^{-11} \sqrt{\frac{T}{100 \mathrm{~K}}}\left(\frac{n_{\mathrm{H}}}{100 \mathrm{~cm}^{-3}}\right) \times J \times F
$$

for the damping contribution, and

$$
\begin{aligned}
\left(\tau^{-1} \Delta J\right)= & 3.9 \times 10^{-6}\left(\frac{T}{100 \mathrm{~K}}\right)^{\frac{3}{2}} \\
& \times\left(\frac{n_{\mathrm{H}}}{100 \mathrm{~cm}^{-3}}\right)\left(\frac{N_{\mathrm{C}}}{50}\right)^{2} \times \frac{1}{J} \times G
\end{aligned}
$$

for the exciting contribution, where $F$ and $G$ are normalized rates defined in the Appendix B of DL98. We use the formalism of Bakes \& Tielens (1994) to estimate the average charge of PAHs of a given size.

\section{D.2. Rocket effect}

Ejection of $\mathrm{H}$ or $\mathrm{H}_{2}$ from the edges of PAHs may yield a significant rotational excitation if it occurs asymmetrically, thus generating a systematic torque by the rocket effect (Rouan et al. 1992). We note below $E_{\text {ej }}$ the kinetic energy of the ejected fragment. In the case of $\mathrm{H}_{2}$, this will happen if this molecule forms on preferential sites by chemisorption, and if the distribution of these sites on the PAH is asymmetric as a result of dehydrogenation. We calculate the change of $J$ assuming that $\mathrm{H}_{2}$ molecules are ejected from the edge of the PAH with a cosine law:

$$
\begin{aligned}
\left(\tau^{-1} \Delta J\right)_{\mathrm{H}_{2}}= & 3.4 \times 10^{-8}\left(\frac{N_{\mathrm{C}}}{50}\right)^{3 / 2}\left(\frac{n_{\mathrm{H}}}{100 \mathrm{~cm}^{-3}}\right) \\
& \times \sqrt{\frac{T}{100 \mathrm{~K}}} \sqrt{\frac{E_{\mathrm{ej}}}{1.5 \mathrm{eV}}}\left(1-f_{\mathrm{H}}\right) \mathrm{s}^{-1} .
\end{aligned}
$$

The above numerical values are based on the following assumptions: (i) all molecular hydrogen is formed on PAHs with $[\mathrm{C} / \mathrm{H}]_{\mathrm{PAH}}=4 \times 10^{-5}$, a $\mathrm{H}_{2}$ formation rate $R_{f}=3 \times$ $10^{-17}(T / 70 \mathrm{~K})^{1 / 2} \mathrm{~cm}^{3} \mathrm{~s}^{-1}$ (Jura 1975) and $E_{\mathrm{ej}}=1.5 \mathrm{eV}$; (ii) the distribution of formation sites has an asymmetry of 1 site and we assume that the site in excess is always at the same location on the molecule; (iii) we neglect the influence of crossover events that may reduce the angular momentum (Lazarian \& Draine 1999). With all these assumptions, the spin rate due to $\mathrm{H}_{2}$ formation estimated here is an upper limit. This rate, however, remains small compared to the other gas-grains processes.

\section{D.3. Photoelectric effect}

Stellar UV photons can pull out electrons from grains. These photoelectrons carry away a significant kinetic energy $(\sim 1 \mathrm{eV})$ that heats the interstellar gas and impulses grain rotation. If we 


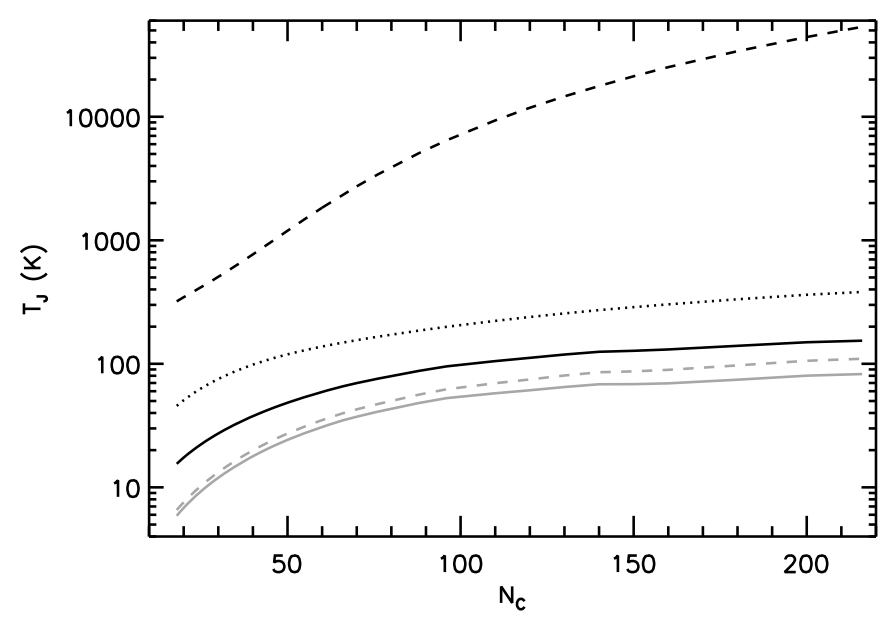

Fig. E.1. Rotational temperature for PAH cations as a function of their size for several insterstellar environments: CNM (black line), WNM (gray dashed line), WIM (gray line), MC (black dotted line) and the Orion Bar (black dashed line).

assume that the photoelectrons leave the grain surface with a cosine law distribution, we have

$\left(\tau^{-1} \Delta J\right)_{\mathrm{pe}}=\tau_{\mathrm{pe}}^{-1} \times 0.15 \sqrt{\frac{N_{\mathrm{C}}}{50}} \sqrt{\frac{E_{\mathrm{e}-}}{1 \mathrm{eV}}} \mathrm{s}^{-1}$

where $\tau_{\mathrm{pe}}^{-1}$ is the rate of photoelectrons ejections calculated with the formalism of Bakes \& Tielens (1994) $\left(\tau_{\mathrm{pe}}^{-1}=1.6 \times 10^{-8} \mathrm{~s}^{-1}\right.$ for $N_{\mathrm{C}}=50$ in the DIM).

\section{Appendix E: Rotational temperature}

A rotational temperature $T_{J}$ can be defined from the Maxwell distribution of angular momentum, $n(J)=n_{0} \exp \left(-J^{2} / J_{0}^{2}\right)$ :

$T_{J}(\mathrm{~K})=\frac{B J_{0}^{2}}{k_{B}}=10 \times N_{\mathrm{C}}^{-2} J_{0}^{2}$.

Figure E.1 shows this temperature as a function of PAH size for several interstellar environments. We see that $T_{J}$ is subthermal in the case of the DIM, and suprathermal for MCs and the Orion Bar. These results are in good agreement with Rouan et al. (1997). The rotational temperature is a relevant parameter in the study of the width of PAH vibrational or electronic transitions. The latter have been proposed as the origin of some unidentified diffuse interstellar bands (DIBs).

\section{References}

Ali-Haïmoud, Y., Hirata, C. M., \& Dickinson, C. 2009, MNRAS, 395, 1055 Allamandola, L. J., Tielens, A. G. G. M., \& Barker, J. R. 1985, ApJ, 290, L25 Andersson, B.-G., Wannier, P. G., Moriarty-Schieven, G. H., \& Bakker, E. J. 2000, AJ, 119, 1325
Baer, T., \& Hase, W. 1996, Unimolecular reaction dynamics: theory and experiments (Oxford university press)

Bakes, E. L. O., \& Tielens, A. G. G. M. 1994, ApJ, 427, 822

Beyer, T., \& Swinehart, D. 1973, Comm. of the ACM, 16

Boulanger, F. 2000, in ESA SP-455: ISO Beyond Point Sources: Studies of Extended Infrared Emission, ed. R. J. Laureijs, K. Leech, \& M. F. Kessler, 3

Casassus, S., Cabrera, G. F., Förster, F., et al. 2006, ApJ, 639, 951

Compiègne, M., Abergel, A., Verstraete, L., \& Habart, E. 2008, A\&A, 491, 797 Davies, R. D., Dickinson, C., Banday, A. J., et al. 2006, MNRAS, 370, 1125 de Oliveira-Costa, A., Tegmark, M., Finkbeiner, D. P., et al. 2002, ApJ, 567, 363

Draine, B. T., \& Lazarian, A. 1998, ApJ, 508, 157

Draine, B. T., \& Li, A. 2001, ApJ, 551, 807

Dupac, X., Bernard, J.-P., Boudet, N., et al. 2003, A\&A, 404, L11

Ferland, G. J., Korista, K. T., Verner, D. A., et al. 1998, PASP, 110, 761

Flagey, N., Boulanger, F., Verstraete, L., et al. 2006, A\&A, 453, 969

Herzberg, G. 1968a,b, Diatomic Molecules (a), IR and Raman Spectra of Polyatomic Molecules (b) (Van Norstand)

Joblin, C., Leger, A., \& Martin, P. 1992, ApJ, 393, L79

Joblin, C., Boissel, P., Leger, A., D’Hendecourt, L., \& Defourneau, D. 1995, A\&A, 299, 835

Joblin, C., \& Mulas, G. 2008, in School on dust, Les Houches, ed. F. Boulanger (EAS Publication Series)

Jura, M. 1975, ApJ, 197, 575

Kim, H.-S., \& Saykally, R. J. 2002, ApJS, 143, 455

Kim, S.-H., Martin, P. G., \& Hendry, P. D. 1994, ApJ, 422, 164

Lagache, G. 2003, A\&A, 405, 813

Lazarian, A., \& Draine, B. T. 1999, ApJ, 516, L37

Le Page, V., Snow, T. P., \& Bierbaum, V. M. 2003, ApJ, 584, 316

Léger, A., \& Puget, J. L. 1984, A\&A, 137, L5

Léger, A., D’Hendecourt, L., \& Defourneau, D. 1989, A\&A, 216, 148

Leitch, E. M., Readhead, A. C. S., Pearson, T. J., \& Myers, S. T. 1997, ApJ, 486, L23

Lovas, F. J., McMahon, R. J., Grabow, J.-U., et al. 2005, J. Am. Chem. Soc., 127,4345

Malloci, G., Joblin, C., \& Mulas, G. 2007, Chem. Phys., 332, 353

Mathis, J. S., Rumpl, W., \& Nordsieck, K. H. 1977, ApJ, 217, 425

Miville-Deschênes, M.-A., Ysard, N., Lavabre, A., et al. 2008, A\&A, 490, 1093

Mulas, G. 1998, A\&A, 338, 243

Mulas, G., Malloci, G., Joblin, C., \& Toublanc, D. 2006, A\&A, 460, 93

Omont, A. 1986, A\&A, 164, 159

Pech, C., Joblin, C., \& Boissel, P. 2002, A\&A, 388, 639

Peeters, E., Hony, S., Van Kerckhoven, C., et al. 2002, A\&A, 390, 1089

Peeters, E., Allamandola, L. J., Bauschlicher, Jr., C. W., et al. 2004a, ApJ, 604, 252

Peeters, E., Mattioda, A. L., Hudgins, D. M., \& Allamandola, L. J. 2004b, ApJ, 617, L65

Rapacioli, M., Joblin, C., \& Boissel, P. 2005, A\&A, 429, 193

Ridge, N. A., Schnee, S. L., Goodman, A. A., \& Foster, J. B. 2006, ApJ, 643, 932

Roche, P. F., Lucas, P. W., \& Geballe, T. R. 1996, MNRAS, 281, L25

Rouan, D., Leger, A., Omont, A., \& Giard, M. 1992, A\&A, 253, 498

Rouan, D., Leger, A., \& Le Coupanec, P. 1997, A\&A, 324, 661

Schutte, W. A., Tielens, A. G. G. M., \& Allamandola, L. J. 1993, ApJ, 415, 397

Smith, J. D. T., Dale, D. A., Armus, L., et al. 2004, ApJS, 154, 199

Smith, J. D. T., Draine, B. T., Dale, D. A., et al. 2007, ApJ, 656, 770

Socrates, G. 2001, IR and Raman characteristic group frequencies: Tables and charts (John Wiley \& Sons)

Townes, C., \& Schawlow, A. 1975, Microwave Spectroscopy (Dover Publications)

Tripathi, A. K., \& Sahasrabudhe, A., Mitra, R., et al. 2001, Phys. Chem. Chem. Phys., 3, 449

Verstraete, L., \& Léger, A. 1992, A\&A, 266, 513

Verstraete, L., Pech, C., Moutou, C., et al. 2001, A\&A, 372, 981

Watson, R. A., Rebolo, R., Rubiño-Martín, J. A., et al. 2005, ApJ, 624, L89

Werner, M. W., Uchida, K. I., Sellgren, K., et al. 2004, ApJS, 154, 309

Ysard, N., Miville-Deschênes, M. A., \& Verstraete, L. 2010, A\&A, 509, L1 